\title{
A presentation for the baseleaf preserving mapping class group of the punctured solenoid
}

\author{
SYLVAIN BONNOT \\ ROBERT C PENNER \\ DRAGOMIR ŠARIĆ
}

\begin{abstract}
We give a presentation for the baseleaf preserving mapping class group $\operatorname{MCG}(\mathcal{H})$ of the punctured solenoid $\mathcal{H}$. The generators for our presentation were introduced previously, and several relations among them were derived. In addition, we show that $\operatorname{MCG}(\mathcal{H})$ has no non-trivial central elements. Our main tool is a new complex of triangulations of the disk upon which $\operatorname{MCG}(\mathcal{H})$ acts.
\end{abstract}

57M99; 20F65

\section{Introduction}

This note continues the investigation (begun in Penner-Šaric [14]) of the baseleaf preserving mapping class group $\operatorname{MCG}(\mathcal{H})$ for the punctured solenoid $\mathcal{H}$. Our main result is a presentation for $\operatorname{MCG}(\mathcal{H})$. The punctured solenoid $\mathcal{H}$ is an inverse limit of the system of all finite unbranched covers of a finite punctured surface of negative Euler characteristic, and its baseleaf preserving mapping class group $\mathrm{MCG}(\mathcal{H})$ consists of all homotopy classes of appropriate self-maps of $\mathcal{H}$ which preserve a distinguished leaf. (See Section 2 or [14] for more details.)

Sullivan [15] introduced the universal hyperbolic solenoid (as the inverse limit of finite unbranched covers of a compact base surface) and the commensurator group of the fundamental group of the base surface as the natural mapping class group of the universal hyperbolic solenoid. Our definitions from [14] of the punctured solenoid $\mathcal{H}$ and its baseleaf preserving mapping class group are analogous to [15] in the presence of the punctures on the base surface.

We recall that the Ehrenpreis conjecture states that any two compact Riemann surfaces have almost conformal finite unbranched covers of the same genus. Sullivan observed (Nag-Sullivan [9]) that the Ehrenpreis conjecture is equivalent to the statement that the mapping class group of the universal hyperbolic solenoid has dense orbits in the Teichmüller space of the universal hyperbolic solenoid. One can state an analogous 
conjecture (to the Ehrenpreis conjecture) for the punctured surfaces. Namely, given any $\epsilon>0$ and given any two negative Euler characteristic finite punctured surfaces there exist their finite unbranched covers that are $\epsilon$ close to being conformal, ie $(1+\epsilon)-$ quasiconformal. This is equivalent to the statement that the (baseleaf preserving) mapping class group $\operatorname{MCG}(\mathcal{H})$ has dense orbits in the Teichmüller space $T(\mathcal{H})$ of the punctured solenoid $\mathcal{H}$. This motivates the study of $\operatorname{MCG}(\mathcal{H})$.

Another motivation (for the study of $\operatorname{MCG}(\mathcal{H})$ ) is that the baseleaf preserving mapping class group $\mathrm{MCG}(\mathcal{H})$ is a large subgroup of the studied commensurator group $\operatorname{Comm}\left(F_{2}\right)$ of the free group $F_{2}$ on two generators (for the definition, see Section 2). Namely, if we identify $F_{2}$ with the once punctured torus group $G$, then $\operatorname{MCG}(\mathcal{H})$ is the subgroup of $\operatorname{Comm}(G)$ which preserves peripheral elements, ie, preserves parabolic elements.

The universal hyperbolic solenoid $\mathcal{H}$ is a topological space locally homeomorphic to the product of a 2-disk and a Cantor set with one topological end. The path components of the punctured solenoid $\mathcal{H}$, called leaves, are homeomorphic to the unit disk $\mathbf{D}$ and the baseleaf is a fixed distinguished leaf. Since $\operatorname{MCG}(\mathcal{H})$ preserves the baseleaf, which is dense in $\mathcal{H}$, it is enough to analyse the action of $\operatorname{MCG}(\mathcal{H})$ on the baseleaf (which is conformally equivalent to the unit disk $\mathbf{D})$. It was shown in [14] that $\operatorname{MCG}(\mathcal{H})$ is isomorphic to the subgroup of quasisymmetric maps of $S^{1}$ which conjugate finite index subgroups of $\mathrm{PSL}_{2}(\mathbb{Z})$ onto possibly different finite index subgroups of $\mathrm{PSL}_{2}(\mathbb{Z})$ (see Odden [11] and Penner-Šarić [14]). We use this identification through the paper without further mentioning.

Given an ideal triangulation of the unit disk D (ie, the baseleaf) which is invariant under a finite index subgroup $K$ of $\mathrm{PSL}_{2}(\mathbb{Z})$ and a specified edge of the triangulation, there are two adjacent triangles which together form a "neighboring" quadrilateral. We may replace the specified edge of this quadrilateral by its other diagonal, and performing this modification for each edge in the $K$-orbit of the specified edge, we define the $K$-equivariant Whitehead move. The resulting ideal triangulation is also invariant under $K$. A Whitehead homeomorphism of $S^{1}$ is obtained by mapping the ideal triangulation of the unit disk $\mathbf{D}$ invariant under a finite index subgroup $K$ of $\mathrm{PSL}_{2}(\mathbb{Z})$ onto its image under a Whitehead move. It is shown in [14] that the Whitehead homeomorphisms together with $\mathrm{PSL}_{2}(\mathbb{Z})$ generate the baseleaf preserving mapping class group $\operatorname{MCG}(\mathcal{H})$ (see Section 2 or [14] for more details). In [14], four relations among Whitehead homeomorphisms are identified and three of them arise in our presentation (see Theorem 1.2(c)).

We first introduce the triangulation complex $\mathcal{X}$ for the punctured solenoid $\mathcal{H}$. The vertices of $\mathcal{X}$ are TLC tesselations, ie ideal triangulations of $\mathbf{D}$ invariant under some 
finite index subgroup of $\operatorname{PSL}_{2}(\mathbb{Z})$. The basepoint $\tau_{*}$ of the triangulation complex $\mathcal{X}$ is the Farey tesselation $\tau_{*}:=\mathcal{F}$. Two vertices of $\mathcal{X}$ are joined by an edge if they differ by a Whitehead move.

There are several types of two-cells in $\mathcal{X}$ : two edges of a triangulation may have disjoint neighboring quadrilaterals, in which case there is a two-cell corresponding to commutativity of their associated Whitehead moves; the two edges may have neighboring quadrilaterals which share a triangle, in which case there is a two-cell corresponding to the pentagon relation; or a single Whitehead move equivariant for a finite index subgroup $K<\mathrm{PSL}_{2}(\mathbb{Z})$ may be written as the finite composition of Whitehead moves equivariant for a subgroup of $K$ of finite index. (The two-cells are described more precisely in Section 3).

Theorem 1.1 The triangulation complex $\mathcal{X}$ is connected and simply connected.

The action of $\operatorname{MCG}(\mathcal{H})$ on the triangulation complex $\mathcal{X}$ is evidently cellular. Furthermore (see [14]), there is only one orbit of vertices in $\mathcal{X}$, and the isotropy group of a vertex $\tau$, ie, its stabilizer $\Gamma(\tau)$, is a conjugate of $\operatorname{PSL}_{2}(\mathbb{Z})$. In fact, the stabilizer $\Gamma\left(\tau_{*}\right)$ of the basepoint $\tau_{*}$ is $\mathrm{PSL}_{2}(\mathbb{Z})$. Together with further analysis of the isotropy group $\Gamma(E)$ of an unoriented edge $E$, standard techniques (Brown [2]) allow us to derive a presentation of $\mathrm{MCG}(\mathcal{H})$. To simplify this presentation, we actually choose a larger set of generators for $\operatorname{MCG}(\mathcal{H})$, namely, we take as generators all Whitehead moves starting from the basepoint of $\mathcal{X}$. (This is a smaller set of generators than in [14] but larger than necessary.) We denote by $\mathcal{E}^{+}$the set of edges of $\mathcal{X}$ which contain the basepoint and are not inverted by an element of $\operatorname{MCG}(\mathcal{H})$, and by $\mathcal{E}^{-}$the set of edges which contain the basepoint and are inverted by an element of $\operatorname{MCG}(\mathcal{H})$. It is necessary to fix one Whitehead homeomorphism $g_{E}$ for each edge $E \in \mathcal{E}^{+}$in a consistent way. (See Section 4 regarding this choice.) Let $\mathcal{E}^{ \pm}=\mathcal{E}^{+} \sqcup \mathcal{E}^{-}$denote the set of unoriented edges, and let $\Gamma^{+}(E)$ denote the subgroup of $\Gamma(E)$ which does not invert the edge $E \in \mathcal{E}^{-}$. Our main theorem is as follows.

Theorem 1.2 The (baseleaf preserving) mapping class group $\mathrm{MCG}(\mathcal{H})$ is generated by the isotropy subgroup $\mathrm{PSL}_{2}(\mathbb{Z})$ of the basepoint $\tau_{*} \in \mathcal{X}$, the isotropy subgroups $\Gamma(E)$ for $E \in \mathcal{E}^{ \pm}$, and by the elements $g_{E}$ for $E \in \mathcal{E}^{+}$. The following relations on these generators give a complete presentation of $\operatorname{MCG}(\mathcal{H})$ :

(a) The inclusions of $\Gamma(E)$ into $\operatorname{PSL}_{2}(\mathbb{Z})$, for $E \in \mathcal{E}^{+}$, are given by $\Gamma(E)=K^{\prime}$, where the terminal endpoint of $E$ is a TLC tesselation invariant under a finite index subgroup $K^{\prime}<\mathrm{PSL}_{2}(\mathbb{Z})$. 
(b) The inclusions of $\Gamma^{+}(E)$ into $\mathrm{PSL}_{2}(\mathbb{Z})$, for $E \in \mathcal{E}^{-}$, are given by $\Gamma(E)=K^{\prime}$, where the terminal endpoint of $E$ is a TLC tesselation invariant under a finite index subgroup $K^{\prime}<\mathrm{PSL}_{2}(\mathbb{Z})$.

(c) The relations introduced by the boundary edge-paths of two-cells in $\mathcal{F}$ given by the equations (4-1), (4-2), (4-3), (4-4), (4-5), (4-6) and (4-7) in Section 4.

(d) The redundancy relations: for any two edges $E$ and $E^{\prime}$ in $\mathcal{E}^{ \pm}$and for any $\gamma \in \mathrm{PSL}_{2}(\mathbb{Z})$ such that $\gamma(E)=E^{\prime}$, we get the relation

$$
g_{E^{\prime}} \circ \gamma^{\prime}=\gamma \circ g_{E},
$$

where $\gamma^{\prime}$ is the unique element of $\operatorname{PSL}_{2}(\mathbb{Z})$ that satisfies $\gamma^{\prime}\left(e_{0}\right)=e_{1}^{\prime}$ with $e_{1}^{\prime}=g_{E^{\prime}}^{-1}\left(\gamma\left(e_{0}\right)\right)$ and $e_{0}=(-1,1)$ is the oriented geodesic in $\mathbf{D}$ with the initial point $-1 \in S^{1}$ and the terminal point $1 \in S^{1}$ which is the standard distinguished oriented edge of $\mathcal{F}$.

It is well-known that the mapping class group of a Riemann surface of finite type has trivial center provided the genus is at least three, and we obtain the analogous result for $\operatorname{MCG}(\mathcal{H})$.

Theorem 1.3 The baseleaf preserving mapping class group $\operatorname{MCG}(\mathcal{H})$ of the punctured solenoid $\mathcal{H}$ has trivial center.

Define $\mathcal{Y}=\mathcal{X} / \operatorname{MCG}(\mathcal{H})$ and let $\mathcal{N}$ be the subgroup of $\operatorname{MCG}(\mathcal{H})$ generated by all elements which fix a point in $\mathcal{X}$. By a standard result of Armstrong [1], we get the following Theorem.

Theorem 1.4 The topological fundamental group of $\mathcal{Y}=\mathcal{X} / \operatorname{MCG}(\mathcal{H})$ is given by

$$
\pi_{1}(\mathcal{Y})=\operatorname{MCG}(\mathcal{H}) / \mathcal{N}
$$

Acknowledgements We are grateful to John Milnor for useful comments. We also thank the referee whose careful reading has led to many improvements in our paper.

\section{Preliminaries}

Fix a finite punctured surface $S$ (the base surface) with negative Euler characteristic and empty boundary, and consider the system of all finite unbranched covers of $S$. There is a partial ordering on the covers as follows. If one cover $\pi_{1}$ can be factored as the composition of two covers $\pi_{1}=\pi \circ \pi_{2}$, where $\pi, \pi_{2}$ are also finite unbranched covers, then $\pi_{1} \geq \pi_{2}$. The system of covers is inverse directed, and there is thus an inverse limit. 
Definition 2.1 The punctured solenoid $\mathcal{H}$ is the inverse limit of the system of finite unbranched covers of a punctured surface without boundary and with negative Euler characteristic.

The inverse limit does not depend on the base surface as long as it is of negative Euler characteristic (see Odden [11] and Penner-Šarić [14]). The punctured solenoid $\mathcal{H}$ is locally homeomorphic to a disk times a Cantor set. Each path component is called a leaf, and each leaf is homeomorphic to the unit disk. The punctured solenoid $\mathcal{H}$ has uncountably many leaves, each of which is dense in $\mathcal{H}$. If we require in the above definition of $\mathcal{H}$ that each punctured surfaces and each covering map is pointed, we obtain a distinguished point, called the basepoint of $\mathcal{H}$. The leaf containing the basepoint is called the baseleaf. The punctured solenoid $\mathcal{H}$ is a non-compact topological space with one end, which is homeomorphic to a horoball times a Cantor set modulo the continuous action of a countable group. For more details see [14].

Definition 2.2 The baseleaf preserving mapping class group $\mathrm{MCG}(\mathcal{H})$ of the punctured solenoid $\mathcal{H}$ is the group of isotopy classes all self-homeomorphisms of $\mathcal{H}$ which preserve the baseleaf, which are leafwise quasiconformal and which vary continuously for the transverse variation in the Teichmüller metric on quasiconformal maps on the global leaves.

Remark Let $f$ be a quasiconformal map of the unit disk D. The Beltrami dilatation of $f$ is an essentially bounded measurable function on $\mathbf{D}$ given by $\mu(f):=\frac{\bar{\partial} f}{\partial f}$. The Teichmüller metric on the space of quasiconformal maps of $\mathbf{D}$ is given by the essential supremum norm on the corresponding vector space of Beltrami dilatations. Note that the Teichmüller metric is a pseudometric.

Remark The mapping class group of a compact surface is defined as the group of homeomorphisms of the surface modulo the normal subgroup of the homeomorphisms homotopic to the identity. It is a standard fact that any homeomorphism of a compact surface is homotopic to a quasiconformal map using for example the barycentric extension (see Douady-Earle [3]). In the case of Riemann surfaces of infinite geometric type, an arbitrary homeomorphism is not homotopic to a quasiconformal map. In this case, the definition of the mapping class group starts with the quasiconformal maps. In the case of the punctured solenoid $\mathcal{H}$, each leaf is conformal to the unit disk which is a geometrically infinite Riemann surface. Therefore, it is convenient to start with the quasiconformal maps. Further, the Teichmüller metric on quasiconformal maps is an appropriate way to make sense of the continuity for the transverse variation. 
The restriction of an element of $\operatorname{MCG}(\mathcal{H})$ to the baseleaf gives a quasiconformal homeomorphism of the unit disk D (upon fixing an identification of the baseleaf with D) up to isotopy. Thus, an element of $\operatorname{MCG}(\mathcal{H})$ determines a well-defined quasisymmetric homeomorphism of $S^{1}$, and we shall thus identify $\operatorname{MCG}(\mathcal{H})$ with an appropriate group of quasisymmetric maps. (See Theorem 2.8.)

Definition 2.3 The commensurator group $\operatorname{Comm}(G)$ of a group $G$ consists of equivalence classes of isomorphisms of finite index subgroups of $G$, where two isomorphisms are equivalent if they agree on a finite index subgroup in the intersection of their domains.

In the literature, the commensurator group $\operatorname{Comm}(G)$ is also called the virtual automorphism group of $G$. It appears under this name in Odden [11], Nag-Sullivan [9] and Penner-Šarić [14].

We will need the following result, which appears as [14, Theorem 7.2] and which is itself an adaptation of the main theorem in the article [11] by Odden.

Theorem 2.4 (Penner-Šarić [14]) The baseleaf preserving mapping class group $\operatorname{MCG}(\mathcal{H})$ is isomorphic to a proper subgroup of the commensurator group $\operatorname{Comm}\left(F_{2}\right)$ of the free group $F_{2}$ on two generators. Namely, $\operatorname{MCG}(\mathcal{H})$ is isomorphic to the subgroup of $\operatorname{Comm}\left(F_{2}\right)$ consisting of all elements which preserve the peripheral elements under some fixed identification $F_{2} \equiv G$, where $G<\mathrm{PSL}_{2}(\mathbb{Z})$ is a group uniformizing the once-punctured torus.

In fact, it is convenient in the definition of the punctured solenoid $\mathcal{H}$ to fix the base surface to be the Modular once-punctured torus $\mathbf{D} / G$, where $G<\mathrm{PSL}_{2}$ ( $\mathbb{Z}$ ). Given an isomorphism of two finite index subgroups $K, H$ of $G$ which preserves peripheral elements, there exists a unique quasisymmetric map of $S^{1}$ which conjugates $K$ onto $H$. (To see this, note that the fact that there is a peripheral preserving isomorphism $\psi$ between $K$ and $H$ implies that the surfaces $\mathbf{D} / K$ and $\mathbf{D} / H$ have the same signature by the classification of the finite punctured surfaces. Thus there exists a homeomorphism $h$ between the surfaces $\mathbf{D} / H$ and $\mathbf{D} / K$ which lifts to a homeomorphism of $\mathbf{D}$ which conjugates $H$ onto $K$. We denote by $h_{*}$ the induced isomorphism between $H$ and $K$. By composing $\psi$ with the induced isomorphism $h_{*}$ we obtain an isomorphism of $K$ which preserves the peripheral elements. By the Baer-Dehn-Nielsen theorem in the version for the punctured surfaces, there exists a homeomorphism $g$ of $\mathbf{D} / K$ which induces $h_{*} \circ \psi$. Thus $\psi$ is induced $\tilde{h}^{-1} \circ \widetilde{g}$ with possibly post-composing by an element of $H$, where $\widetilde{g}, \tilde{h}$ are lifts to $\mathbf{D}$ of $g, h$ respectively.) Thus, by the previous 
theorem, we may consider $\mathrm{MCG}(\mathcal{H})$ as a group of quasisymmetric maps of $S^{1}$ which conjugate one finite index subgroup of $\mathrm{PSL}_{2}(\mathbb{Z})$ onto another.

We recall that the decorated Teichmüller space $\widetilde{T}(\mathcal{H})$ of the punctured solenoid $\mathcal{H}$ consists of all hyperbolic metrics on $\mathcal{H}$ together with an assignment of horoballs at the "punctures" (see [14] for more details). Each horoball is identified with a single point in the light cone of the Minkowskii three space corresponding to the leaf "containing" the puncture. By taking the bending locus of the boundary of the convex hull of the points in the light cone for each leaf, we assign a leafwise geodesic lamination on $\mathcal{H}$. Then $\widetilde{T}(\mathcal{H})$ is partitioned into sets according to the bending leafwise geodesic lamination of the convex hull construction [14] (see Epstein-Penner [4] and Penner $[12 ; 13]$ for convex hull construction). When the bending locus is a triangulation on each leaf, then it is locally constant in the transverse direction, and the action of the baseleaf preserving mapping class group $\operatorname{MCG}(\mathcal{H})$ is transitive on this subspace of $\tilde{T}(\mathcal{H})$ (see [14] for details). The above makes it convenient to consider the action of $\operatorname{MCG}(\mathcal{H})$ on the ideal triangulations of the baseleaf (ie, the unit disk $\mathbf{D}$ ) arising by restrictions (to the baseleaf) of the triangulations of $\mathcal{H}$.

Definition 2.5 A transversely locally constant (TLC) tesselation $\tau$ of the unit disk D is a lift to $\mathbf{D}$ of an ideal triangulation of some punctured surface $\mathbf{D} / K$ of finite type, ie, an ideal triangulation of $\mathbf{D}$ invariant under a finite index subgroup $K$ of $\mathrm{PSL}_{2}(\mathbb{Z})$, where the ideal points of the tesselation agree with $\overline{\mathbb{Q}} \subset S^{1}$.

A particularly important example of a TLC tesselation is the Farey tesselation $\tau_{*}:=\mathcal{F}$ (see Figure 1 or, for example, [13] or [14]), which is invariant under the group $\operatorname{PSL}_{2}(\mathbb{Z})$.

Let $K$ be a finite index subgroup of $\mathrm{PSL}_{2}(\mathbb{Z})$ and let $\tau$ be a $K$-invariant TLC tesselation of D. A characteristic map for $\tau$ is a homeomorphism $h: S^{1} \rightarrow S^{1}$ such that $h\left(\tau_{*}\right)=\tau$ (see [13, Theorem 6.3 and 6.4]). The map $h=h(\tau, e)$ is completely determined by specifying an oriented edge $e \in \tau$, namely, the standard oriented edge $e_{0}=(-1,1)$ in $\tau_{*}$ is mapped onto $e$, the triangle to the left or right of $e_{0}$ in $\tau_{*}$ is mapped to the triangle to the left or right, respectively, of $e$ in $\tau$, and so on. Note that any two characteristic maps for $\tau$ differ by pre-composition with an element of $\mathrm{PSL}_{2}(\mathbb{Z})$.

Theorem 2.6 (Penner-Šarić [14, Lemma 7.5]) The characteristic map $h=h(\tau, e)$ for a $K$-invariant TLC tesselation $\tau$ conjugates a finite index subgroup $H$ of $\mathrm{PSL}_{2}(\mathbb{Z})$ onto $K$.

Note that a characteristic map necessarily conjugates peripherals into peripherals because it is a homeomorphisms of $S^{1}$, and so a characteristic map for a TLC tesselation lies in $\operatorname{MCG}(\mathcal{H})$. 


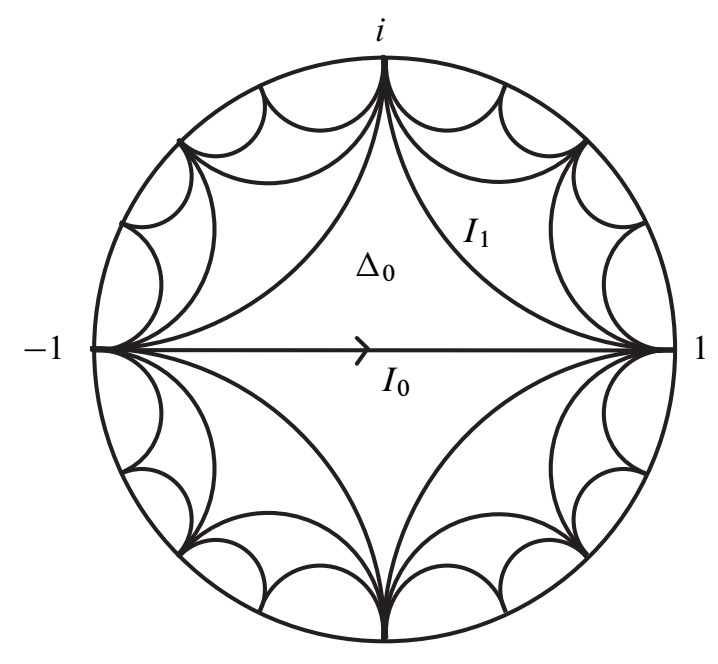

Figure 1: The Farey tesselation $F ; I_{0}=(-1,1) ; I_{1}=(1, i)$.

Let $\tau$ be a $K$-invariant TLC tesselation of $\mathbf{D}$ with a distinguished oriented edge $e$; we allow for the possibility that $\tau$ is invariant under a larger subgroup of $\operatorname{PSL}_{2}(\mathbb{Z})$. Fix an edge $f \in \tau$. We form a new $K$-invariant TLC tesselation $\tau^{\prime}$ by replacing each $\gamma(f)$, for $\gamma \in K$, by $\gamma\left(f^{\prime}\right)$, where $f^{\prime}$ is the diagonal of the unique ideal quadrilateral in $(\mathbf{D}-\tau) \cup\{f\}$ different from $f$. We say that $\tau^{\prime}$ is obtained from $\tau$ by performing a Whitehead move along $K\{f\}$ (see Figure 2). If $e \notin K\{f\}$ then we let $e$ be the distinguished oriented edge in $\tau^{\prime}$ as well; if $e=\gamma(f)$ for some $\gamma \in K$, then we let $e^{\prime}=\gamma\left(f^{\prime}\right)$ be the distinguished oriented edge for $\tau^{\prime}$, where $e^{\prime}$ is given the orientation such that the tangent vectors to $e$ and $e^{\prime}$ at their intersection point comprise a positively oriented basis for the oriented disk $\mathbf{D}$.

Definition 2.7 Let $\tau$ be a $K$-invariant TLC tesselation of $\mathbf{D}$ with a distinguished oriented edge $e$. The Whitehead homeomorphism for $\tau$ and $e$ is

$$
k(\tau, e)=h\left(\tau^{\prime}, e^{\prime}\right) \circ h(\tau, e)^{-1},
$$

where $\left(\tau^{\prime}, e^{\prime}\right)$ arises from $(\tau, e)$ under the Whitehead move and $h\left(\tau^{\prime}, e^{\prime}\right), h(\tau, e)$ are the characteristic maps.

We will also use the notation $k(\tau, K, e)$ whenever we need to put emphasis on the group $K$. A Whitehead homeomorphism lies in $\operatorname{MCG}(\mathcal{H})$ since it is the composition of two elements of the group $\operatorname{MCG}(\mathcal{H})$ by Theorem 2.6. 

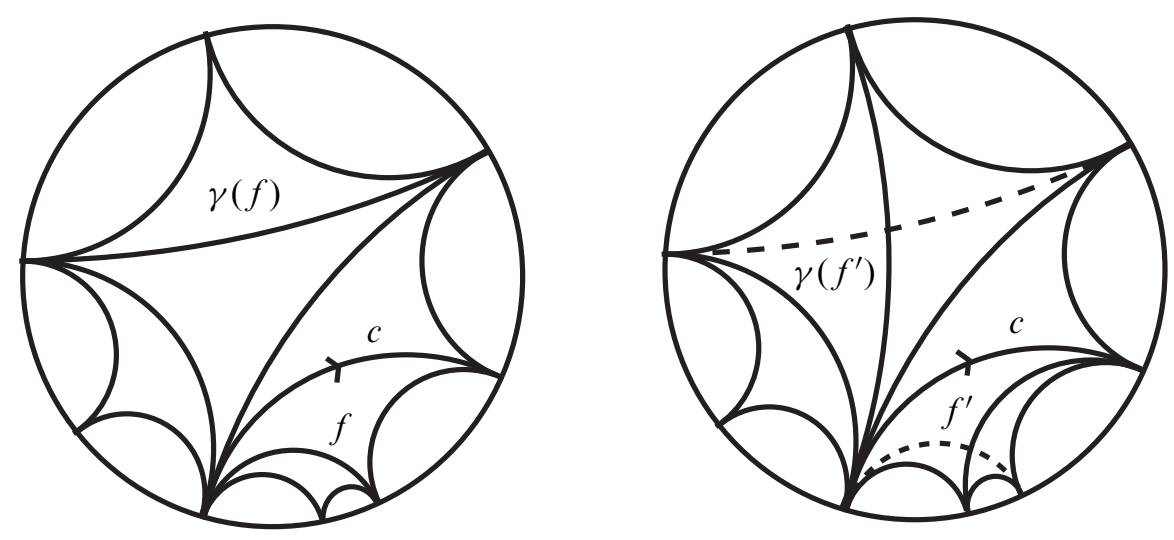

Figure 2: Whitehead move along $K\{f\}$.

Theorem 2.8 (Penner-Šarić [14]) The baseleaf preserving mapping class group $\operatorname{MCG}(\mathcal{H})$ of the punctured solenoid $\mathcal{H}$ is generated by $\operatorname{PSL}_{2}(\mathbb{Z})$ and by Whitehead homeomorphisms for all TLC tesselations. In addition, $\operatorname{MCG}(\mathcal{H})$ acts transitively on the set of all TLC tesselations of the unit disk $\mathbf{D}$.

\section{The triangulation complex}

We introduce a two-complex $\mathcal{X}$ associated to TLC tesselations of $\mathbf{D}$. This complex $\mathcal{X}$ is an adaptation to our situation of the two-skeleton of the complex dual to the cell decomposition of the decorated Teichmüller space introduced by Penner [12] and Harer [5]. On the other hand, $\mathcal{X}$ is analogous to the complex of cut systems of Hatcher and Thurston [7] in that $\operatorname{MCG}(\mathcal{H})$ acts transitively on its vertices.

We begin the definition of $\mathcal{X}$ by giving its vertices. A vertex of the triangulation complex $\mathcal{X}$ is a TLC tesselation of the unit disk $\mathbf{D}$. The basepoint of $\mathcal{X}$ is the Farey tesselation $\tau_{*}$. A characteristic map between any two TLC tesselations is an element of $\operatorname{MCG}(\mathcal{H})$ [14, Lemma 7.5], and so $\operatorname{MCG}(\mathcal{H})$ acts transitively on vertices of $\mathcal{X}$.

We next introduce edges of $\mathcal{X}$ with one endpoint at the basepoint $\tau_{*}$. An unordered pair of vertices $\left\{\tau, \tau_{*}\right\}$ determines an edge in $\mathcal{X}$ if $\tau$ can be obtained from $\tau_{*}$ by a single Whitehead move, ie, $\tau$ is obtained from $\tau_{*}$ by replacing an orbit $K\{f\}$ of an edge $f$ in $\tau_{*}$ by the orbit $K\left\{f^{\prime}\right\}$, where $K$ is torsion-free and of finite index in 
$\operatorname{PSL}_{2}(\mathbb{Z})$, and $f^{\prime}$ is the diagonal of the quadrilateral in $\left(\mathbf{D}-\tau_{*}\right) \cup\{f\}$ different from $f$.

More generally and by definition, an unordered pair of vertices $\left\{\tau_{1}, \tau_{2}\right\}$ determines an edge of $\mathcal{X}$ if $\left\{\tau_{1}, \tau_{2}\right\}$ is the image by an element of $\operatorname{MCG}(\mathcal{H})$ of an edge $\left\{\tau_{*}, \tau\right\}$ defined above. In particular, this implies that if a TLC tesselation $\tau_{2}$ is obtained by performing a $K$-invariant Whitehead move on a TLC tesselation $\tau_{1}$ then $\left\{\tau_{1}, \tau_{2}\right\}$ is an edge in $\mathcal{X}$. To see this, take a characteristic map $h$ for $\tau_{1}$ (ie, $h$ is a homeomorphism of $S^{1}$ such that $\left.h\left(\tau_{*}\right)=\tau_{1}\right)$ and consider $\left\{\tau_{*}, h^{-1}\left(\tau_{2}\right)\right\}$. By definition, we know that our two tesselations $\tau_{1}$ and $\tau_{2}$ differ only in the $K$ orbit of the diagonals of an ideal rectangle. Now, the characteristic map $h$ conjugates a finite index subgroup $H$ of $\operatorname{PSL}_{2}(\mathbb{Z})$ onto $K\left[14\right.$, Lemma 7.5], so $h^{-1}\left(\tau_{1}\right)=\tau_{*}$ and $h^{-1}\left(\tau_{2}\right)$ differ only in that they have different diagonals on an $H$-orbit of a rectangle. It follows that $\left\{\tau_{*}, h^{-1}\left(\tau_{2}\right)\right\}$ is an edge corresponding to an $H$-invariant Whitehead move, ie, $\left\{\tau_{1}, \tau_{2}\right\}=h\left(\left\{\tau_{*}, h^{-1}\left(\tau_{2}\right)\right\}\right)$ is an edge in $\mathcal{X}$.

However, there are edges which appear away from the basepoint $\tau_{*}$ that do not correspond to Whitehead moves. It is enough to take $h \in \operatorname{MCG}(\mathcal{H})$ which conjugates a maximal torsion free finite index subgroup $K$ of $\operatorname{PSL}_{2}(\mathbb{Z})$ onto another finite index subgroup and a Whitehead move on $\tau_{*}$ for a torsion free group $K_{1}<\mathrm{PSL}_{2}(\mathbb{Z})$ such that $\left[K_{1}: K\right]>1$. If $E=\left\{\tau_{*}, \tau\right\}$ is the edge corresponding to the above Whitehead move, then the edge $h(E)=\left\{h\left(\tau_{*}\right), h(\tau)\right\}$ does not correspond to a Whitehead move. These edges can be described as corresponding to a generalized Whitehead move invariant under a conjugate $h K_{1} h^{-1} \nless \mathrm{PSL}_{2}(\mathbb{Z})$ by a characteristic map of a finite index subgroup $K_{1}$ of $\mathrm{PSL}_{2}(\mathbb{Z})$. However, $h K_{1} h^{-1} \cap K_{1}$ is of finite index in $K_{1}$. Note that the set of edges in $\mathcal{X}$ is invariant under the action of $\operatorname{MCG}(\mathcal{H})$ by construction. This completes the definition of the one-skeleton of $\mathcal{X}$.

We introduce two-cells of $\mathcal{X}$ by first defining those that have one vertex at the basepoint $\tau_{*}$. Let $K$ be a torsion-free finite index subgroup of $\mathrm{PSL}_{2}(\mathbb{Z})$. There are three types of two-cells.

Pentagon Suppose that $K$ is of index at least 9 , ie, $\tau_{*} / K$ is a triangulation of D/ $K$ which has at least three complementary ideal triangles. Any three adjacent complementary triangles form a pentagon on $\mathbf{D} / K$ whose boundary sides are possibly identified in pairs. Let $e_{1}$ and $e_{2}$ be two representatives in $\tau_{*}$ of the diagonals of a pentagon on $\mathbf{D} / K$ which share an ideal point. The sequence of five Whitehead homeomorphisms $k\left(\tau_{*}, K, e_{1}\right), k\left(\tau_{1}, K, e_{2}\right), k\left(\tau_{2}, K, e_{1}^{\prime}\right), k\left(\tau_{3}, K, e_{2}^{\prime}\right)$ and $k\left(\tau_{4}, K, e_{1}^{\prime \prime}\right)$ defines a closed edge-path in $\mathcal{X}$ based at $\tau_{*}$, where $e_{1}^{\prime}$ is the new edge corresponding to $e_{1}$ under the Whitehead move for $\tau_{*}$ along $K\left\{e_{1}\right\}, e_{2}^{\prime}$ is the new edge corresponding to $e_{2}$ under the Whitehead move for $\tau_{1}=h\left(K, e_{1}\right)\left(\tau_{*}\right)$ along $K\left\{e_{2}\right\}, e_{1}^{\prime \prime}$ is the new 
edge corresponding to $e_{1}^{\prime}$ under the Whitehead move for $\tau_{2}=k\left(\tau_{1}, K, e_{2}\right)\left(\tau_{1}\right)$ along $K\left\{e_{1}^{\prime}\right\}$ and $e_{2}^{\prime \prime}$ is the new edge (which is equal to $e_{1}$ ) corresponding to $e_{2}^{\prime}$ under the Whitehead move for $\tau_{3}=k\left(\tau_{2}, K, e_{2}^{\prime}\right)\left(\tau_{2}\right)$ along $K\left\{e_{2}^{\prime}\right\}$ (see the related illustration). We add a two-cell in $\mathcal{X}$ whose boundary is this closed edge-path of length five starting and ending at the basepoint $\tau_{*}$ and call this two-cell a pentagon at the basepoint $\tau_{*}$.

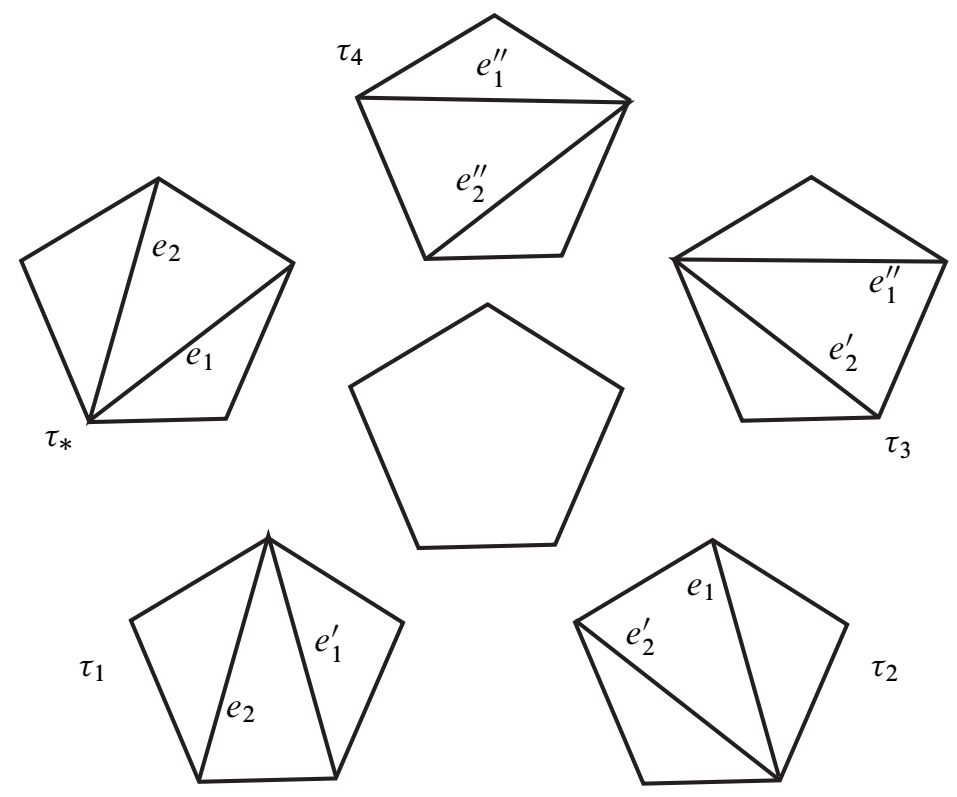

Figure 3: Pentagon

Square Let $K$ be a torsion-free finite index subgroup of $\mathrm{PSL}_{2}(\mathbb{Z})$ such that the triangulation $\tau_{*} / K$ of $\mathbf{D} / K$ has two edges which do not lie in the boundary of a common complementary triangle. Let $e_{1}$ and $e_{2}$ be two lifts to $\tau_{*}$ of the two nonadjacent edges. Consider the closed edge-path of length four given by Whitehead homeomorphisms $k\left(\tau_{*}, K, e_{1}\right), k\left(\tau_{1}, K, e_{2}\right), k\left(\tau_{2}, K, e_{1}^{\prime}\right)$ and $k\left(\tau_{3}, K, e_{2}^{\prime}\right)$, where $e_{1}^{\prime}$ corresponds to $e_{1}$ under the Whitehead move on $\tau_{*}$ along $K\left\{e_{1}\right\}$ and $e_{2}^{\prime}$ corresponds to $e_{2}$ under the Whitehead move on $\tau_{1}$ along $K\left\{e_{2}\right\}$. We add a two-cell to $\mathcal{X}$ with boundary equal to the above edge-path of length four and call it a square cell at the basepoint $\tau_{*}$.

Coset Suppose $e \in \tau_{*}$ and let $H$ be a finite index subgroup of $K$. The orbit $K\{e\}$ is canonically decomposed into finitely many orbits $H\left\{e_{1}\right\}, H\left\{e_{2}\right\}, \ldots, H\left\{e_{n}\right\}$, where $e_{1}=e, e_{2}, \ldots, e_{n} \in K\{e\}$ and $n=[K: H]$. Let $f$ be the other diagonal in the unique ideal quadrilateral in $\left(\mathbf{D}-\tau_{*}\right) \cup\{e\}$ and let $f_{1}, f_{2}, \ldots, f_{n} \in K\{f\}$ be the altered edges 


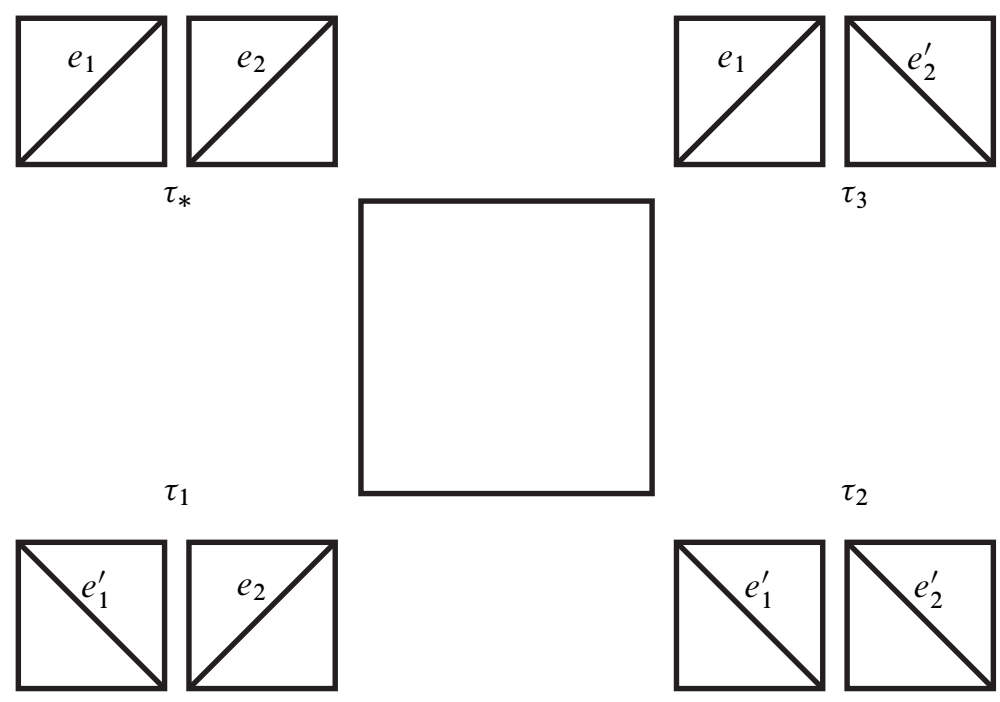

Figure 4: Square

corresponding to $e_{1}, e_{2}, \ldots, e_{n}$. Consider a finite edge-path based at $\tau_{*}$ consisting of the Whitehead homeomorphisms

$$
k\left(\tau_{*}, K, e_{1}\right)=h(K, e), k\left(\tau_{1}, H, f_{2}\right), k\left(\tau_{2}, H, f_{3}\right), \ldots, k\left(\tau_{n-1}, H, f_{n}\right)
$$

corresponding to the tesselations

$$
\begin{aligned}
\tau_{*}, \tau_{1} & =h(K, e)\left(\tau_{*}\right), \tau_{2}=k\left(\tau_{1}, H, f_{2}\right)\left(\tau_{1}\right), \ldots, \tau_{n-1}=k\left(\tau_{n-2}, H, f_{n-1}\right)\left(\tau_{n-2}\right) \\
\tau_{n} & =k\left(\tau_{n-1}, H, f_{n}\right)\left(\tau_{n-1}\right)=\tau_{*} .
\end{aligned}
$$

We add a two-cell to $\mathcal{X}$ whose boundary is this edge-path and call it the coset cell at the basepoint. Note that a different ordering of $f_{1}, \ldots, f_{n}$ gives a different edge-path and hence a different coset cell. In fact, there are $n$ ! corresponding coset cells when $[K: H]=n$. The edge $\left\{\tau_{*}, \tau_{1}\right\}$ is called a long edge, and all other edges are called short edges corresponding to this coset cell.

Note that all two-cells introduced above have their boundaries given by compositions of Whitehead moves invariant under subgroups of $\mathrm{PSL}_{2}(\mathbb{Z})$ as opposed to more general edges in $\mathcal{X}$ where moves are only conjugate to Whitehead moves invariant under subgroups of $\mathrm{PSL}_{2}(\mathbb{Z})$.

To complete the definition of $\mathcal{X}$, an arbitrary two-cell in $\mathcal{X}$ is the image under $\operatorname{MCG}(\mathcal{H})$ of a two-cell at the basepoint. If $h \in \operatorname{MCG}(\mathcal{H})$ and $P$ is a two-cell based at $\tau_{*}$, then we say that $h(P)$ is based at $\tau:=h\left(\tau_{*}\right)$. Note that closed edge-paths based at $\tau_{*}$ are mapped to closed edge-paths, and hence the boundaries of two-cells are well defined. 
The boundary of a pentagon or a square two-cell based at a tesselation $\tau \neq \tau_{*}$ each of whose edges is a Whitehead move invariant with respect to a fixed finite index subgroup $K$ of $\mathrm{PSL}_{2}(\mathbb{Z})$ (or equivalently, whose vertices are TLC tesselations invariant under $K$ ) is likewise the boundary of a two-cell. Furthermore, the boundary of a coset two-cell starting at $\tau \neq \tau_{*}$ whose initial vertex is invariant under $K$ and whose other vertices are invariant under a subgroup $K_{1}<K$ of finite index is the image of the boundary of a coset two-cell based at $\tau_{*}$ by simply noting that a characteristic map which sends $\tau_{*}$ onto $\tau$ conjugates $H_{1}<H$ onto $K_{1}<K$ where $H<\operatorname{PSL}_{2}(\mathbb{Z})$.

By construction, the set of two-cells in $\mathcal{X}$ is invariant under $\operatorname{MCG}(\mathcal{H})$, and $\operatorname{MCG}(\mathcal{H})$ consequently acts cellularly on the two-complex $\mathcal{X}$.

We claim that a pentagon or a square two-cell $P$ based at $\tau \neq \tau_{*}$ with one vertex at the basepoint $\tau_{*}$ has all edges given by Whitehead moves invariant under a fixed finite index subgroup $K$ of $\operatorname{PSL}_{2}(\mathbb{Z})$. Since any characteristic map $h$ of $\tau_{*}$ onto the base vertex $\tau$ of the two-cell $P$ conjugates a finite index subgroup $H<\operatorname{PSL}_{2}(\mathbb{Z})$ onto a finite index subgroup $K<\mathrm{PSL}_{2}(\mathbb{Z})$ (under which $\tau$ is invariant), it follows that a two-cell (pentagon or square) $P^{\prime}$ invariant under $H$ and based at $\tau_{*}$ is mapped by $h$ onto the above two-cell $P$ based at $\tau$ whose vertices are invariant under $K$. Moreover, a coset two-cell $P$ which is based at $\tau \neq \tau_{*}$ whose initial vertex is $\tau_{*}$ does not necessarily have edges arising from Whitehead moves invariant under $K_{1}<K$. Let $P^{\prime}$ be a coset cell based at $\tau_{*}$ such that $h\left(P^{\prime}\right)=P$. In fact, if the image under $h$ of the long edge of $P^{\prime}$ is not incident on $\tau_{*}$, then it is represented by a generalized Whitehead move (invariant under a conjugate by $h$ of a subgroup of $\operatorname{PSL}_{2}(\mathbb{Z})$ which is not itself a group of Möbius transformations).

Theorem 1.1 The triangulation complex $\mathcal{X}$ is connected and simply connected.

Proof We first prove that $\mathcal{X}$ is connected by showing that any vertex $\tau$ can be connected to the basepoint $\tau_{*}$ by a finite edge-path. Let $K$ be a finite index subgroup of $\operatorname{PSL}_{2}(\mathbb{Z})$ under which $\tau$ is invariant. Thus, $\tau / K$ and $\tau_{*} / K$ are two tesselations of a punctured surface $\mathbf{D} / K$. By results of Penner [12, Proposition 7.1] (or Harer [5, Theorem 1.1] or Hatcher [6, Main corollary]), there is a sequence of Whitehead moves on $\mathbf{D} / K$ which transforms $\tau_{*} / K$ into $\tau / K$. The lifts of the Whitehead moves on $\mathbf{D} / K$ to $\mathbf{D}$ are TLC Whitehead moves on $\mathbf{D}$ and they provide an edge-path from $\tau_{*}$ to $\tau$ in $\mathcal{X}$. This establishes that $\mathcal{X}$ is connected.

It remains to show that $\mathcal{X}$ is simply connected. In the literature, the triangulation complex of a finite punctured surface is often referred to as the arc complex. We recall a result of Harer [5, Theorem 1.3] or Penner [12, Theorem 5.5] for triangulations of punctured surfaces: the set of top-dimensional simplices of the triangulation complex 
of a finite punctured surface consists of ideal triangulations, the codimension-one simplices are ideal triangulations with one ideal geodesic erased, the codimension-two simplices are ideal triangulations with two ideal geodesics erased, etc. The main fact is that the triangulation complex of the finite surface minus simplices which are given by decompositions of the surface, where at least one complementary component is not topologically a disc is homeomorphic to the decorated Teichmüller space of the punctured surface. In particular, the triangulation complex for a punctured surface is contractible.

Consider a closed edge-path $\alpha$ in the triangulation complex $\mathcal{X}$ for the punctured solenoid. It is possible that an edge $E$ in the path $\alpha$ is given by a generalized Whitehead move, ie, the two tesselations at the endpoints of $E$ are invariant under $h K h^{-1}$, where $K$ is a finite index subgroup of $\operatorname{PSL}_{2}(\mathbb{Z})$ and $h \in \operatorname{MCG}(\mathcal{H})$. Since $h \in \operatorname{MCG}(\mathcal{H})$, there exists $H_{1}, H_{2}<\mathrm{PSL}_{2}(\mathbb{Z})$ of finite index such that $H_{2}=h H_{1} h^{-1}$. Thus, $H_{1} \cap K=: K_{1}$ is of finite index in $K$, and we consider a coset two-cell corresponding to the groups $K_{1}<K$ with long edge $h^{-1}(E)$. The edge $h^{-1}(E)$ is homotopic modulo its endpoints to the path of short edges in the coset two-cell, where each vertex is invariant under $K_{1}$. The image under $h$ is a coset two-cell with long edge corresponding to $h K h^{-1}$ and short edges corresponding to $h K_{1} h^{-1}<\mathrm{PSL}_{2}(\mathbb{Z})$. Thus, we can replace the long edge invariant under $h K h^{-1}$, which is not a subgroup of $\operatorname{PSL}_{2}(\mathbb{Z})$, by the homotopic edge-path invariant under $h K_{1} h^{-1}<H_{2}<\mathrm{PSL}_{2}(\mathbb{Z})$. We may therefore replace $\alpha$ by an edge-path $\alpha^{\prime}$ each of whose edges corresponds to a Whitehead move invariant under a finite index subgroup of $\mathrm{PSL}_{2}(\mathbb{Z})$ using only coset two-cells.

Let $K_{1}, K_{2}, \ldots, K_{n}$ be finite index subgroups of $\mathrm{PSL}_{2}(\mathbb{Z})$ which correspond to invariant Whitehead moves defining the edges of $\alpha^{\prime}$. Using coset two-cells corresponding to each $K_{i}$, we first homotope the above edge-path $\alpha^{\prime}$ into a closed edge-path $\alpha^{\prime \prime}$ where each edge corresponds to an invariant Whitehead move with respect to a single finite index subgroup $K:=K_{1} \cap K_{2} \cap \cdots \cap K_{n}$. The new edge-path $\alpha^{\prime \prime}$ invariant under $K$ in the triangulation complex $\mathcal{X}$ of the punctured solenoid can be represented by a closed path $\gamma$ in the above triangulation complex of a finite surface $\mathbf{D} / K$. (Recall that $\mathcal{X}$ is an extension of the dual of the triangulation complex of finite surface $\mathbf{D} / K$.) The path $\gamma$ starts and ends in the top-dimensional simplex which corresponds to the triangulation of $\mathbf{D} / K$ obtained by projecting the TLC tesselation of $\mathbf{D}$ defining the basepoint of $\alpha^{\prime \prime}$ onto $\mathbf{D} / K$. Furthermore, $\gamma$ crosses transversely codimension-one simplices of the triangulation complex of $\mathbf{D} / K$ corresponding to each edge in $\alpha^{\prime \prime}$, and it enters each top-dimensional simplex which corresponds to a vertex of $\alpha^{\prime \prime}$ in the given order.

Since the triangulation complex for punctured surface $\mathbf{D} / K$ is simply connected ([5, Theorem 1.3] or [12], as cited above), there exists a homotopy of $\gamma$ into the trivial path 
which transversely crosses codimension-two cells. The number of times the homotopy crosses codimension-two cells is finite, and it is possible to choose a homotopy which does not intersect simplices of codimension greater than two. For each intersection point of the homotopy with a codimension-two simplex, there is a corresponding two-cell in $\mathcal{X}$ because two-cells corresponding to ideal triangulations of the surface with two edges erased lift to ideal triangulations of $\mathbf{D}$ with orbits of two edges erased such that each complementary region is finite sided. This exactly correspond to two-cells (either pentagon or square) in $\mathcal{X}$. Thus, the homotopy for $\gamma$ gives a homotopy between $\alpha^{\prime \prime}$ and the trivial path in $\mathcal{X}$, and $\mathcal{X}$ is therefore simply connected.

In the spirit of Ivanov's work [8], we may ask the following question.

Question Is the group of automorphisms $\operatorname{Aut}(\mathcal{X})$ of the triangulation complex $\mathcal{X}$ isomorphic to the (extended) baseleaf preserving baseleaf preserving mapping class group?

\section{Presentation of $\operatorname{MCG}(\mathcal{H})$}

Applying a general theorem of Brown [2, Theorem 1] to the action of $\operatorname{MCG}(\mathcal{H})$ on $\mathcal{X}$, we give a presentation for the baseleaf preserving mapping class group $\operatorname{MCG}(\mathcal{H})$ of the punctured solenoid $\mathcal{H}$.

Brown's Theorem [2, Theorem 1] Let $G$ be an arbitrary group acting on a connected non-empty $C W$-complex $X$. Brown's theorem requires a certain number of choices, given as follows (see Brown [2] for details). We will review these choices in greater details during the discussion preceding our Theorem 1.2.

(a) Choose an orientation $P$ (which is by definition a set $P$ of oriented edges that are preserved with their orientations by $G$ );

(b) Choose a tree of representatives $T$, and let $V$ be its set of vertices;

(c) Choose a set $E^{+}$of representatives for $P \bmod G$, such that each edge $e \in E^{+}$ has its initial vertex $o(e) \in V$ and such that each 1 -cell of $T$ (with its chosen orientation) is in $E^{+}$. Choose a set $E^{-}$of representatives mod $G$ for the edges of $X$ which are inverted under the action, again with $o(e) \in V$ for each $e \in E^{-}$, and let $\Sigma^{-}$be the corresponding set of $1-$ cells of $X$;

(d) For each $e \in E^{+}$let $w=w(e)$ be the unique element of $V$ which is equivalent $\bmod G$ to the end point $t(e)$, and choose an element $g_{e} \in G$ such that $t(e)=$ $g_{e} w$. 
(e) Choose a set $F$ of representatives $\bmod G$ for the 2-cells of $X$, and choose an attaching map for each $\tau \in F$;

(f) For each $\tau \in F$, choose a word $r_{\tau}$ that represents the relation associated to the boundary of the cell $\tau$.

Theorem 1 (Brown [2]) Suppose $X$ is simply-connected. Then $G$ is generated by the isotropy subgroups $G_{v}(v \in V)$, the isotropy subgroups $G_{\sigma}\left(\sigma \in \Sigma^{-}\right)$, and the elements $g_{e}\left(e \in E^{+}\right)$, subject to the following relations.

(i) $g_{e}=1$ if $e$ is an edge of $T$.

(ii) $g_{e}^{-1} i_{e}(g) g_{e}=c_{e}(g)$ for any $e \in E^{+}$and $g \in G_{e}$, where $i_{e}$ is the inclusion $G_{e} \rightarrow G_{o(e)}$ and $c_{e}: G_{e} \rightarrow G_{w(e)}$ is as in (d) above. [Thus both sides of the 'relation' are words in the given generators of $G$.]

(iii) $i_{e}(g)=j_{e}(g)$ for any $e \in E^{-}$and $g \in G_{e}$, where $i_{e}: G_{e} \rightarrow G_{o(e)}$ and $j_{e}: G_{e} \rightarrow G_{\sigma}$ are inclusions, $\sigma$ being the 1-cell underlying $e$.

(iv) $r_{\tau}=1$ (or, equivalently, $\hat{g}_{\tau}=g_{\tau}$ ) for any $\tau \in F$.

From [14, Lemma 7.3] we know that $\mathrm{PSL}_{2}(\mathbb{Z})$ is the isotropy group of the basepoint $\tau_{*} \in \mathcal{X}$. From [14, Theorem 7.6], we know that $\operatorname{MCG}(\mathcal{H})$ acts transitively on the vertices of $\mathcal{X}$, and that an arbitrary vertex $\tau \in \mathcal{X}$ has isotropy group $h \mathrm{PSL}_{2}(\mathbb{Z}) h^{-1}$, where the characteristic map $h: \tau_{*} \mapsto \tau$ lies in $\operatorname{MCG}(\mathcal{H})$, and that $h \operatorname{PSL}_{2}(\mathbb{Z}) h^{-1}$ contains a finite index subgroup of $\mathrm{PSL}_{2}(\mathbb{Z})$.

Consider the isotropy group of an edge in $\mathcal{X}$. Since each vertex is mapped to the basepoint $\tau_{*}$, it is enough to consider edges with an endpoint at $\tau_{*}$. The isotropy group of any other edge is the conjugate of the isotropy group of such an edge.

Let $E=\left\{\tau_{*}, \tau\right\}$ be an arbitrary edge of $\mathcal{X}$ with one endpoint at the basepoint $\tau_{*}$ of $\mathcal{X}$. There are two possibilities: either the isotropy group $\Gamma(E)$ of $E$ contains elements which reverse the orientation of $E$ (ie, interchanges $\tau_{*}$ and $\tau$ ), or each element of $\Gamma(E)$ fixes each endpoint of $E$.

Let $\tau$ be obtained by a Whitehead move on $\tau_{*}$ invariant under a torsion-free finite index subgroup $K$ of $\operatorname{PSL}_{2}(\mathbb{Z})$, and let us choose a characteristic map $g: \tau_{*} \mapsto \tau$, where $g \in \operatorname{MCG}(\mathcal{H})$. Denote by $K^{\prime}$ the maximal extension of $K$ in $\operatorname{PSL}_{2}(\mathbb{Z})$ which fixes $\tau$. Then $K^{\prime}$ is a subgroup of the normalizer of $K$ in $\operatorname{PSL}_{2}(\mathbb{Z})$. If $h \in \Gamma(E)$ preserves the orientation of $E$ as above, then $h$ fixes both $\tau_{*}$ and $\tau$. By [14, Lemma 7.3], $h \in$ $\mathrm{PSL}_{2}(\mathbb{Z})$ and similarly $h \in g \mathrm{PSL}_{2}(\mathbb{Z}) g^{-1}$, and so $h \in \mathrm{PSL}_{2}(\mathbb{Z}) \cap g \mathrm{PSL}_{2}(\mathbb{Z}) g^{-1}=K^{\prime}$. It follows that the subgroup $\Gamma^{+}(E)$ of the isotropy group $\Gamma(E)$ of an edge $E$ which consists of elements which do not reverse orientation on $E$ is equal to $K^{\prime}<\mathrm{PSL}_{2}(\mathbb{Z})$. 
If $k \in \Gamma(E)$ reverses orientation of $E=\left\{\tau_{*}, \tau\right\}$, ie, $k\left(\tau_{*}\right)=\tau$ and $k(\tau)=\tau_{*}$, then $k^{2} \in K^{\prime}=\Gamma^{+}(E)$. In particular, $k^{2}$ is a lift of a self-map of the Riemann surface $\mathbf{D} / K$. By Odden [10, Proposition 1.3.6], $k$ is a lift of a self-map of a Riemann surface which finitely covers $\mathbf{D} / K$. We show that $k$ is actually a lift of a self-map of $\mathbf{D} / K$ itself.

Lemma 4.1 Let $\tau$ be the image of $\tau_{*}$ under a $K$-invariant Whitehead move, let $E=\left\{\tau_{*}, \tau\right\}$ be the corresponding edge and let $k \in \Gamma(E)-\Gamma^{+}(E)$. Then $k$ conjugates $K$ onto itself.

Proof The proof proceeds in several steps.

Simplification of the homeomorphism $k$ Since $k$ preserves the union of the two tesselations $\tau, \tau_{*}$, it therefore sends a pair of intersecting edges to a pair of intersecting edges. Fix such an intersecting pair $e \in \tau_{*}$ and $f \in \tau$, and consider the corresponding Whitehead move. Since $k(e) \in K\{f\}$, there exists $\gamma \in K$ such that $(k \circ \gamma)(e)=f$. It is enough to prove the lemma for $k \circ \gamma$ and we continue to denote it by $k$.

Orientation of edges Let us choose an orientation of $e$ and assign an orientation to $f$ such that $k: e \mapsto f$ is orientation-preserving. Assign an orientation to each edge in the orbits of $e$ and $f$ under $K$ as follows. Let $e^{\prime}=\gamma^{\prime}(e)$ for some $\gamma^{\prime} \in K$. Let $\alpha$ be a differentiable arc connecting $e$ to $e^{\prime}$ which transversely crosses the minimal number of edges of $\tau_{*}$. Give the orientation to the curve $\alpha$ such that the tangent vector to $\alpha$ and the tangent vector to $e$ at their point of intersection form a positively oriented basis for the tangent space of $\mathbf{D}$ at the intersection point, and assign an orientation on $e^{\prime}$ such that the tangent vector to $\alpha$ and the tangent vector to $e^{\prime}$ at the intersection point $\alpha \cap e^{\prime}$ form a positively oriented basis to the tangent space. We may assign an orientation to any $f^{\prime}=\gamma^{\prime}(f)$ in a similar fashion.

$k$ preserves the orientation We noted above that $k$ maps the orbit $K\{e\}$ onto $K\{f\}$ without specifying an orientation, and we noted that we may assume that $k(e)=f$ preserving orientation. It is a standard fact that $k: S^{1} \rightarrow S^{1}$ extends to a differentiable self-map $\tilde{k}$ of $\mathbf{D}$ which sends complementary triangles of $\tau_{*}$ onto complementary triangles of $\tau$ [13]. If $\alpha$ is a differentiable path between $e$ and $e^{\prime}$ as above, then $\tilde{k}(\alpha)$ is a differentiable path between $f$ and $f^{\prime \prime}:=k\left(e^{\prime}\right)$ which satisfies the required properties. Note that it is not necessarily true that $f^{\prime}=\gamma^{\prime}(f)$ and $f^{\prime \prime}$ are equal. However, the inductive definition of the characteristic map $k$ immediately implies that $k: e^{\prime} \mapsto f^{\prime \prime}$ is orientation-preserving.

$K$ is orientation-preserving Note that $\gamma \in K$ are covering transformations for the surface $\mathbf{D} / K$. We show that $\gamma: e \mapsto e^{\prime}:=\gamma(e)$ is orientation-preserving, and a 
similar statement for $f$ follows immediately. Denote by $\pi_{K}: \mathbf{D} \rightarrow \mathbf{D} / K$ the universal covering map. Let $\alpha^{\prime}$ be a differentiable curve on $\mathbf{D} / K$ representing the covering transformation $\gamma$ which is transverse to $\pi_{K}(e)$ and crosses the minimal number of edges of $\tau_{*} / K$. We denote by $\alpha$ a part of the lift of $\alpha^{\prime}$ to $\mathbf{D}$ which connects $e$ and $e^{\prime}$, so $\gamma(e \cap \alpha)=e^{\prime} \cap \alpha$. Since $\gamma$ preserves the orientation of $\alpha$, it follows that $\gamma: e \mapsto e^{\prime}$ is orientation-preserving.

$k$ conjugates $K$ onto itself Recall that $k$ conjugates a finite index subgroup $H$ of $\mathrm{PSL}_{2}(\mathbb{Z})$ onto $K$. Since $K$ preserves the orientation of the orbits $K\{e\}$ and $K\{f\}$ in the sense of the previous paragraph and $k$ maps $K\{e\}$ onto $K\{f\}$, it follows that $k$ conjugates the action of $K$ on the orbit $K\{e\}$ onto the action of $K$ on the orbit $K\{f\}$. Since $H$ and $K$ have the same index in $\mathrm{PSL}_{2}(\mathbb{Z})$ it follows that $H=K$.

In the above proof we normalized $k$ such that it maps $e$ to $f$. We do not assume this normalization any more. However, we showed that $k$ necessarily maps $K\{e\}$ onto $K\{f\}$ and that it conjugates $K$ onto itself. Thus, $k$ descends to a self-map $\bar{k}$ of $\mathbf{D} / K$ sending the tesselation $\tau_{*} / K$ onto the tesselation $\tau / K$ and vice versa. Let $\bar{e} \in \tau_{*} / K$ and $\bar{f} \in \tau / K$ be the corresponding edges on $\mathbf{D} / K$ of the orbit $K\{e\} \subset \tau_{*}$ and its corresponding orbit $K\{f\} \in \tau-\tau_{*}$ under the Whitehead move defining the edge $E$.

It follows from the proof above that $\bar{e}$ is necessarily mapped onto $\bar{f}$ by $\bar{k}$, whence $\bar{k}^{2}(\bar{e})=\bar{e}$ and $\bar{k}^{2}(\bar{f})=\bar{f}$ with the orientations of $\bar{e}$ and $\bar{f}$ reversed. This implies that $\bar{k}^{4}(\bar{e})=\bar{e}$ with an orientation of $\bar{e}$ preserved. Since in addition $\bar{k}^{4}\left(\tau_{*} / K\right)=\tau_{*} / K$, we conclude that $\bar{k}^{4}=i d$. This implies that $k$ (after possibly pre-composing by an element of $K$ and for simplicity renaming the composition again by $k$ ) maps $e$ onto $f$ sending $\tau_{*}$ onto $\tau$, and vice versa. Since $k^{2} \in \Gamma(E)$ maps $e$ onto itself by reversing its orientation, we conclude that $k^{2} \in K^{\prime}$ is an involution with fixed point on $e$. Thus,

$$
\Gamma(E)=\left\langle K^{\prime}, k\right\rangle
$$

where $K^{\prime}<\operatorname{PSL}_{2}(\mathbb{Z})$, and $k \in \operatorname{MCG}(\mathcal{H})$ with $k^{2} \in K^{\prime}-K$ and $k^{4}=i d$. In particular, $k^{2}$ is an elliptic involution whose fixed point lies on $e$ and $K^{\prime} \neq K$ if $k^{2}$ is non trivial. Note that any Whitehead move on the once punctured torus can be obtained as a homeomorphism of the torus which interchanges the two tesselations, and hence the corresponding edge is inverted. In the following example we show that edges admit orientation reversing isotropy also for higher genus.

Example 4.2 We give in Figure 1 just one illustrative example of a surface $\mathbf{D} / K$ with a distinguished quadrilateral $Q$, together with a self-homeomorphism performing a Whitehead move on the quadrilateral. In this figure, the homeomorphism $h$ is a rotation by $\pi / 2$ along the horizontal axis. The dots represent the punctures of the surface. 
Now the square of this homeomorphism reverses the orientation of the distinguished diagonal $E$ in the quadrilateral $Q$.
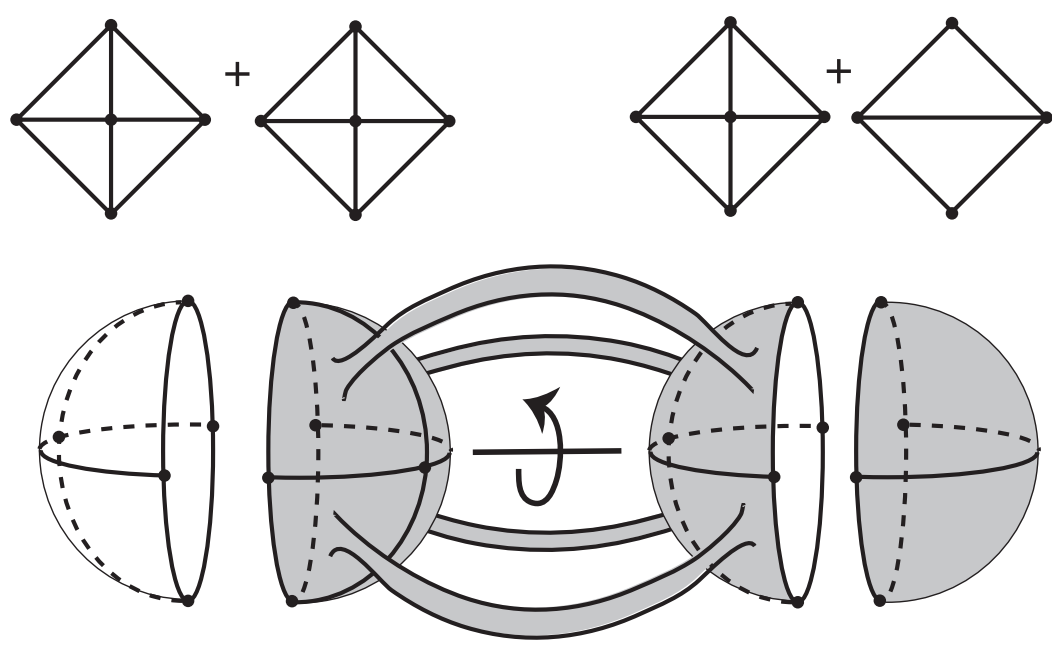

Figure 5: The map $h$ whose square inverts the diagonal $E$.

We also note that there are infinitely many edges $E \in \mathcal{X}$ with $\Gamma(E)-\Gamma^{+}(E)=\varnothing$. This follows from the fact that there are infinitely many Whitehead moves on finite surfaces (whose Euler characteristics are increasing without bound) such that there is no homeomorphism of the surface which maps the starting tesselation onto the ending tesselation and by Lemma 4.1.

Consider a two-cell of $\mathcal{X}$ with one vertex at the basepoint $\tau_{*}$. Recall that for pentagon and square two-cells, each vertex is invariant under a finite index subgroup $K$ of $\mathrm{PSL}_{2}(\mathbb{Z})$, and edges correspond to Whitehead moves invariant under this group $K$. For coset two-cells, either the long edge has $\tau_{*}$ as endpoint, in which case all edges are Whitehead moves invariant under $K$, or the long edge does not have $\tau_{*}$ as endpoint, in which case the long edge is given by a generalized Whitehead move.

We may now apply Brown's theorem [2, Theorem 1] to obtain a presentation of the baseleaf preserving mapping class group $\operatorname{MCG}(\mathcal{H})$ since it acts cellularly on the connected and simply connected triangulation complex $\mathcal{X}$ with a single vertex orbit. In fact, we shall introduce a somewhat larger set of generators than necessary for the application of Brown's theorem in order to obtain a simpler presentation.

It is a standard fact (which follows from Tietze's Theorem for instance) that for a given presentation, if one adds extra generators, then an equivalent presentation arises 
by expressing the new generators in terms of the old as new relations. One can in effect replace any occurrence of a subsequence of old generators in the old relations by new generators, in order to presumably simplify the presentation. We shall ultimately give the presentation of an abstract group $\mathcal{G}$, which is equivalent in this sense to the presentation of the group in Brown's Theorem.

Description of the set of edges $\mathcal{E}^{+}$Notice that the set of edges of $\mathcal{X}$ that are not inverted by the action of $\operatorname{MCG}(\mathcal{H})$ can be oriented consistently for the action of $\operatorname{MCG}(\mathcal{H})$. We fix one such orientation on each such edge.

Let $\mathcal{E}^{+}$be the set of edges which are not inverted by the action of $\operatorname{MCG}(\mathcal{H})$ that have initial point $\tau_{*}$. If $E=\left(\tau_{*}, \tau\right) \in \mathcal{E}^{+}$then $\tau$ is invariant under a finite index subgroup $K$ of $\mathrm{PSL}_{2}(\mathbb{Z})$. By Lemma 4.1 and the subsequent discussion, there is no homeomorphism $f: \mathbf{D} / K \rightarrow \mathbf{D} / K$ such that $f\left(\tau_{*} / K\right)=\tau / K$ and $f(\tau / K)=\tau_{*} / K$. The elements of $\mathcal{E}^{+}$therefore are obtained by taking all finite index torsion-free subgroups of $\mathrm{PSL}_{2}(\mathbb{Z})$ and performing all possible Whitehead moves on $\tau_{*}$ invariant under the chosen groups, where the Farey tesselation $\tau_{*}$ and the image tesselation satisfy the additional property of not being mapped onto each other by a single map conjugating the group onto itself. The images of $\tau_{*}$ under the Whitehead moves are the terminal vertices of edges in $\mathcal{E}^{+}$. For any such $E=\left(\tau_{*}, \tau\right) \in \mathcal{E}^{+}$, we fix the characteristic map $g_{E} \in \operatorname{MCG}(\mathcal{H})$ such that $g_{E}\left(\tau_{*}\right)=\tau$ and the standard distinguished oriented edge $e_{0}=(-1,1)$ of $\tau_{*}$ is mapped to either itself if the Whitehead move is not along an orbit of $e_{0}$, or it is mapped onto $f_{0}=(-i, i)$ if the Whitehead move is along an orbit of $e_{0}$. The characteristic map is uniquely determined by these conditions, and we fix this choice $g_{E}$. (Notice that $\mathcal{E}^{+}$is larger than necessary, since it is enough to take only the edges corresponding to representatives of conjugacy classes in $\mathrm{PSL}_{2}(\mathbb{Z})$ of finite index subgroups. However, this larger set simplifies the presentation, and not much is lost because both sets are infinite.)

Description of the set of edges $\mathcal{E}^{-}$Let $\mathcal{E}^{-}$denote the set of inverted edges with initial point $\tau_{*}$. By Lemma 4.1, an edge $E=\left(\tau_{*}, \tau\right)$ is inverted if there exists $k: S^{1} \rightarrow S^{1}$ such that $k\left(\tau_{*}\right)=\tau, k(\tau)=\tau_{*}$ and $k H k^{-1}=H$, where $\tau$ is invariant under a torsion-free finite index subgroup $H$ of $\operatorname{PSL}_{2}(\mathbb{Z})$. The isotropy group $\Gamma(E)$ of the cell underlying $E \in \mathcal{E}^{-}$is the subgroup of $\operatorname{MCG}(\mathcal{H})$ generated by $H^{\prime}$ and $k$, where $H^{\prime}>H$ is the maximal subgroup of $\operatorname{PSL}_{2}(\mathbb{Z})$ under which $\tau$ is invariant, where $k^{2} \in \mathrm{PSL}_{2}(\mathbb{Z})$ and where $k^{4}=i d$. Fix some choice $g_{E}$ of characteristic map associated to $E$ and take $k=g_{E}$. (Again, we take $\mathcal{E}^{-}$larger then necessary for ease in writing down the relations.)

Description of the set $\mathcal{F}$ Denote by $\mathcal{F}$ the set of two-cells of $\mathcal{X}$ based at $\tau_{*}$. This condition implies that for each coset two-cell in $\mathcal{F}$ the initial point of the long edge 
is $\tau_{*}$ and each edge of the cell is consequently obtained by a geometric Whitehead move, ie, one invariant under a subgroup of $\mathrm{PSL}_{2}(\mathbb{Z})$ as opposed to a conjugate of a subgroup of $\mathrm{PSL}_{2}(\mathbb{Z})$. This property also holds for pentagon and square cells in $\mathcal{F}$. The set $\mathcal{F}$ is obtained by taking all torsion-free finite index subgroups of $\operatorname{PSL}_{2}(\mathbb{Z})$ and taking all possible pentagon, square and coset edge-paths in $\mathcal{X}$ corresponding to the chosen groups.

Note that a single choice of group for coset two-cells gives countably many coset cells because there are countably many finite index subgroups and each finite index subgroup yields finitely many coset cells. In the case of a square or a pentagon cell, a choice of a finite index group determines finitely many cells because there are finitely many edge orbits in $\tau_{*}$ under the group. (Again, we could have taken only representatives of orbits of two-cells based at $\tau_{*}$ together with subsets of $\mathcal{E}^{ \pm}$, but for simplicity later, we have expanded these sets.)

Remark about the choice of generators Let us assume for a moment that we had instead chosen for $\mathcal{E}^{ \pm}$and $\mathcal{F}$ only representatives of classes under the action of $\operatorname{MCG}(\mathcal{H})$. We describe the assignment of a composition of elements in $\operatorname{MCG}(\mathcal{H})$ (depending upon the above choices) to any closed boundary edge-path of a two-cell in $\mathcal{F}$ in order to give a relation corresponding to the two-cell as in Brown's theorem [2]. Given an oriented edge $E=\left(\tau_{*}, \tau\right)$ starting at $\tau_{*}$, we assign to it a chosen element of $g \in \operatorname{MCG}(\mathcal{H})$ such that $\tau=g\left(\tau_{*}\right)$. If $E \in \mathcal{E}^{+}$then set $g:=g_{E}$. If $E \notin \mathcal{E}^{+}$is not inverted by $\operatorname{MCG}(\mathcal{H})$ then $g:=\gamma \circ g_{E^{\prime}}$, where $E^{\prime}=\left(\tau_{*}, \tau^{\prime}\right) \in \mathcal{E}^{+}$, and $\gamma \in \operatorname{PSL}_{2}(\mathbb{Z})$ satisfies $\gamma\left(E^{\prime}\right)=E ; \gamma$ is well-defined up to pre-composition by an element of $\Gamma\left(E^{\prime}\right)$. The two Whitehead moves from $\tau_{*}$ to $\tau$ and $\tau^{\prime}$ determine distinguished oriented edges $e$ and $e^{\prime}$ of $\tau$ and $\tau^{\prime}$, and we choose unique $\gamma \in \mathrm{PSL}_{2}(\mathbb{Z})$ mapping $e^{\prime}$ to $e$. Our choice of $\gamma \circ g_{E}$ is in this case unique. If $E \in \mathcal{E}^{-}$, then set $g:=g_{E} \in \Gamma(E)-\Gamma^{+}(E)$. If $E \notin \mathcal{E}^{-}$is inverted by the action of $\operatorname{MCG}(\mathcal{H})$ then $g:=\gamma \circ g_{E^{\prime}}$, where $E^{\prime} \in \mathcal{E}^{-}$and $\gamma \in \mathrm{PSL}_{2}(\mathbb{Z})$ with $\gamma\left(\tau^{\prime}\right)=\tau$ and $\gamma\left(e^{\prime}\right)=e$. The edge $E$ therefore ends at $g\left(\tau_{*}\right)$, but it seems complicated to explicitly determine $E^{\prime}$ and $\gamma$.

Continuing to assume that we had chosen for $\mathcal{E}^{ \pm}$and $\mathcal{F}$ only representatives of classes under the action of $\operatorname{MCG}(\mathcal{H})$, consider a closed path $\alpha$ of edges in $\mathcal{X}$ based at $\tau_{*}$. Let $\left(E_{1}, E_{2}, \ldots, E_{n}\right)$ be the sequential edges of $\alpha$. Denote by $g_{1}$ the unique element of $\operatorname{MCG}(\mathcal{H})$ chosen for the edge $E_{1}=\left(\tau_{*}, \tau_{1}\right)$ starting at $\tau_{*}$ as above, so $g_{1}\left(\tau_{*}\right)=\tau_{1}$. The edge $E_{2}=\left(\tau_{1}, \tau_{2}\right)$ is therefore of the form $g_{1}\left(E_{2}^{\prime}\right)$ for an edge $E_{2}^{\prime}=\left(\tau_{*}, \tau_{2}^{\prime}\right)$ based at $\tau_{*}$. Denote by $g_{2}$ the unique element of $\operatorname{MCG}(\mathcal{H})$ associated to $E_{2}^{\prime}$ as above, so $g_{1} \circ g_{2}\left(\tau_{*}\right)=\tau_{2}$. This implies that $E_{3}=\left(\tau_{2}, \tau_{3}\right)$ is given by $g_{1} \circ g_{2}\left(E_{3}^{\prime}\right)$, where $E_{3}^{\prime}$ starts at $\tau_{*}$. Take $g_{3} \in \operatorname{MCG}(\mathcal{H})$ associated to the edge $E_{3}^{\prime}$, and continue in this manner until we exhaust all edges of $\alpha$. This yields a composition $g_{1} \circ g_{2} \circ \cdots \circ g_{n}$ in 
terms of generators such that $g_{1} \circ g_{2} \circ \cdots \circ g_{n}\left(\tau_{*}\right)=\tau_{*}$. Thus, there is $\gamma \in \mathrm{PSL}_{2}(\mathbb{Z})$ such that $g_{1} \circ g_{2} \circ \cdots \circ g_{n}=\gamma$, and this is the relation associated with a closed edge path $\alpha$ based at $\tau_{*}$. It seems complicated to determine the maps $g_{i}$ from the given description or to decide which elements $\gamma \in \mathrm{PSL}_{2}(\mathbb{Z})$ arise. However, the choice of $g_{i}$ simplifies if we allow all edges with initial point $\tau_{*}$, and this will require additional relations as discussed before.

From this point on, we go back to our choice of $\mathcal{E}^{ \pm}$to consist of all edges with initial point $\tau_{*}$ and of $\mathcal{F}$ to consists of all two-cells based at $\tau_{*}$.

Relations corresponding to pentagons We describe the relations associated to boundaries of two-cells in $\mathcal{F}$. Let us start with a pentagon two-cell $P$ based at $\tau_{*}$ whose boundary edges are $\left\{E_{1}=\left(\tau_{*}, \tau_{1}\right), E_{2}=\left(\tau_{1}, \tau_{2}\right), \ldots, E_{5}=\left(\tau_{4}, \tau_{*}\right)\right\}$. The pentagon two-cell $P$ is given by changing an orbit of two adjacent edges $e_{1}, e_{2}$ of $\tau_{*}$ under a torsion-free finite index subgroup $K$ of $\mathrm{PSL}_{2}(\mathbb{Z})$ of index at least 9. Assume first that the distinguished oriented edge $e_{0}=(-1,1)$ of $\tau_{*}$ is not an element of the orbit $K\left\{e_{1}, e_{2}\right\}$ and apply the algorithm of Brown to get the edge-path relation, but using our extended set of generators to simplify it. We denote by $g_{i}$ the element of $\operatorname{MCG}(\mathcal{H})$ which corresponds to the edge $E_{i}$. The first edge $E_{1}$ gives $g_{1}:=g_{E_{1}}$, so $g_{1}\left(e_{0}\right)=e_{0}$. We find $g_{2}:=\gamma \circ g_{E_{2}^{\prime \prime}}$, where $E_{2}^{\prime \prime}$ is a representative of the orbit of $\left(\tau_{*}, g_{1}^{-1}\left(\tau_{2}\right)\right)$ and $\gamma\left(E_{2}^{\prime \prime}\right)=\left(\tau_{*}, g_{1}^{-1}\left(\tau_{2}\right)\right)$ is chosen from $\operatorname{PSL}_{2}(\mathbb{Z})$ in a unique way as above (ie, $\left.\gamma\left(e_{0}\right)=e_{0}\right)$. However, since $\mathcal{E}^{+}$consists of all edges starting at $\tau_{*}$ we immediately obtain that $g_{2}:=g_{E_{2}^{\prime}}$, where $E_{2}^{\prime}:=\left(\tau_{*}, g_{1}^{-1}\left(\tau_{2}\right)\right)$. We likewise obtain $g_{i}:=g_{E_{i}^{\prime}}$, for $i=3,4,5$, where $E_{3}^{\prime}:=\left(\tau_{*},\left(g_{1} \circ g_{2}\right)^{-1}\left(\tau_{3}\right)\right), E_{4}^{\prime}:=\left(\tau_{*},\left(g_{1} \circ g_{2} \circ g_{3}\right)^{-1}\left(\tau_{4}\right)\right)$, and $E_{5}^{\prime}:=\left(\tau_{*},\left(g_{1} \circ g_{2} \circ g_{3} \circ g_{4}\right)^{-1}\left(\tau_{*}\right)\right)$. Under our assumption that $e_{0} \notin K\left\{e_{1}, e_{2}\right\}$, we find $g_{i}\left(e_{0}\right)=e_{0}$ for $i=1,2, \ldots, 5$. The relation associated to $P$ is therefore

$$
g_{1} \circ g_{2} \circ \cdots \circ g_{5}=i d \text {. }
$$

On the other hand, now assume $e_{0} \in K\left\{e_{1}\right\}$ and without loss of generality we can assume that $e_{1}=e_{0}$. We choose $g_{i}$ as above and note that $g_{1}:\left(\tau_{*}, e_{0}\right) \mapsto\left(\tau_{1}, e_{0}^{\prime}\right)$, where $e_{0}^{\prime}=(-i, i)$ is the image of $e_{0}=(-1,1)$ under the Whitehead move corresponding to $E_{1}=\left(\tau_{*}, \tau_{1}\right), g_{1} \circ g_{2}:\left(\tau_{*}, e_{0}\right) \mapsto\left(\tau_{2}, e_{0}^{\prime}\right), g_{1} \circ g_{2} \circ g_{3}:\left(\tau_{*}, e_{0}\right) \mapsto\left(\tau_{3}, e_{0}^{\prime \prime}\right)$ where $e_{0}^{\prime \prime}$ is the image of $e_{0}^{\prime}$ under the Whitehead move corresponding to $E_{3}, g_{1} \circ g_{2} \circ$ $g_{3} \circ g_{4}:\left(\tau_{*}, e_{0}\right) \mapsto\left(\tau_{4}, e_{0}^{\prime \prime}\right)$ and $g_{1} \circ g_{2} \circ \cdots \circ g_{5}:\left(\tau_{*}, e_{0}\right) \mapsto\left(\tau_{*}, \bar{e}_{2}\right)$ where $\bar{e}_{2}$ is the oriented edge $e_{2}$ with orientation given such that the terminal point of $e_{0}$ is the initial point of $\bar{e}_{2}$. Denote by $\gamma_{e_{0}, \bar{e}_{2}} \in \mathrm{PSL}_{2}(\mathbb{Z})$ the unique element which maps $e_{0}$ onto $\bar{e}_{2}$ with the given orientations. Thus, $\gamma_{e_{0}, \bar{e}_{2}}$ is the composition of the primitive parabolic element with fixed point at the terminal point of $e_{0}$ which maps $e_{0}$ onto $e_{2}$ and the 
involution which reverses $e_{2}$. We obtain the following relation

$$
g_{1} \circ g_{2} \circ \cdots \circ g_{5}=\gamma_{e_{0}, \bar{e}_{2}} .
$$

When $e_{2}=e_{0}$ the relation is similarly

$$
g_{1} \circ g_{2} \circ \cdots \circ g_{5}=\gamma_{e_{0}, \bar{e}_{1}} \text {. }
$$

Relations corresponding to squares Let $P$ be a square cell in $\mathcal{F}$. Assume that $P$ is obtained by Whitehead moves along the nonadjacent orbits $K\left\{e_{1}\right\}$ and $K\left\{e_{2}\right\}$ of edges $e_{1}, e_{2}$ in $\tau_{*}$, where $K$ is a torsion-free finite index subgroup of $\operatorname{PSL}_{2}(\mathbb{Z})$. If $e_{0} \notin K\left\{e_{1}, e_{2}\right\}$ then

$$
g_{1} \circ \cdots \circ g_{4}=i d,
$$

where $g_{i}$ are chosen as above. If $e_{i}=e_{0}$ then we obtain a relation

$$
g_{1} \circ \cdots \circ g_{4}=s_{e_{0}},
$$

where $s_{e_{0}} \in \mathrm{PSL}_{2}(\mathbb{Z})$ is the involution which reverses $e_{0}$. The proofs of both relations for the square cell $P$ depend upon keeping track of where $e_{0}$ is mapped, and it is sufficiently similar to the pentagon two-cell that we do not repeat it.

Relations corresponding to cosets Let $P \in \mathcal{F}$ be a coset two-cell for the edge $e \in \tau_{*}$ and for the groups $K_{1}<K<\mathrm{PSL}_{2}(\mathbb{Z})$. If $e \neq e_{0}$ then we obtain a relation

$$
g_{1} \circ \cdots \circ g_{n}=i d
$$

where $n=\left[K: K_{1}\right]$ and $g_{i}$ are uniquely chosen as above. Note that a single choice of $K_{1}<K$ gives a decomposition of the orbit $K\{e\}$ into $n$ disjoint coset orbits $K_{1}\left\{e_{1}\right\}, K_{1}\left\{e_{2}\right\}, \ldots, K_{1}\left\{e_{n}\right\}$, where $e_{i} \in K\{e\}$. This gives $n$ ! possible permutations on $K_{1}\left\{e_{1}\right\}, K_{1}\left\{e_{2}\right\}, \ldots, K_{1}\left\{e_{n}\right\}$ which in turn produce $n$ ! coset two-cells with the long edge given by the Whitehead move on $K\{e\}$. Note that $g_{1}=g_{E}$ where $E=\left(\tau_{*}, \tau\right)$ and $\tau$ is the image of the Whitehead move along $K\{e\}$. The other $g_{i}$, for $i=2,3, \ldots, n$, are given by the translation to $\tau_{*}$ of the short edges. If $e=e_{0}$ then we obtain a relation

$$
g_{1} \circ \cdots \circ g_{n}=s_{e_{0}},
$$

where $s_{e_{0}} \in \mathrm{PSL}_{2}(\mathbb{Z})$ is the involution which reverses $e_{0}$ (see the paragraph about Square relations).

The desired group $\mathcal{G}$ is by definition the free product of $\mathrm{PSL}_{2}(\mathbb{Z}), \Gamma(E)=\Gamma^{+}(E)$ for $E \in \mathcal{E}^{+}, \Gamma(E)$ for $E \in \mathcal{E}^{-}$and a free group generated by $g_{E}$ for $E \in \mathcal{E}^{+}$. The 
baseleaf preserving mapping class group $\operatorname{MCG}(\mathcal{H})$ is the quotient of $\mathcal{G}$ by a set of relations as follows.

Theorem 1.2 The baseleaf preserving mapping class group $\operatorname{MCG}(\mathcal{H})$ is generated by the isotropy subgroup $\mathrm{PSL}_{2}(\mathbb{Z})$ of the basepoint $\tau_{*} \in \mathcal{X}$, the isotropy subgroups $\Gamma(E)$ for $E \in \mathcal{E}^{ \pm}$, and by the elements $g_{E}$ for $E \in \mathcal{E}^{+}$. The following relations on these generators give a complete presentation of $\operatorname{MCG}(\mathcal{H})$.

(a) The inclusions of $\Gamma(E)$ into $\mathrm{PSL}_{2}(\mathbb{Z})$, for $E \in \mathcal{E}^{+}$, are given by $\Gamma(E)=K^{\prime}$, where the terminal endpoint of $E$ is invariant under the finite index subgroup $K^{\prime}<\mathrm{PSL}_{2}(\mathbb{Z})$;

(b) The inclusions of $\Gamma^{+}(E)$ into $\mathrm{PSL}_{2}(\mathbb{Z})$, for $E \in \mathcal{E}^{-}$, are given by $\Gamma(E)=K^{\prime}$, where the terminal endpoint of $E$ is invariant under the finite index subgroup $K^{\prime}<\mathrm{PSL}_{2}(\mathbb{Z})$;

(c) The relations introduced by the boundary edge-paths of two-cells in $\mathcal{F}$ given by the equations (4-1), (4-2), (4-3), (4-4), (4-5), (4-6) and (4-7);

(d) The redundancy relations: for any two edges $E$ and $E^{\prime}$ in $\mathcal{E}^{ \pm}$and for any $\gamma \in \mathrm{PSL}_{2}(\mathbb{Z})$ such that $\gamma(E)=E^{\prime}$, we get the relation

$$
g_{E^{\prime}} \circ \gamma^{\prime}=\gamma \circ g_{E}
$$

where $\gamma^{\prime}$ is the unique element of $\operatorname{PSL}_{2}(\mathbb{Z})$ that satisfies $\gamma^{\prime}\left(e_{0}\right)=e_{1}^{\prime}$ with $e_{1}^{\prime}=g_{E^{\prime}}^{-1}\left(\gamma\left(e_{0}\right)\right)$.

Proof The fact about the generators of $\operatorname{MCG}(\mathcal{H})$ follows directly from Brown's theorem [2] and from our choice of $\mathcal{E}^{ \pm}$even larger than necessary. The relations from [2, Theorem 1] are included in our theorem as follows. The relations (i) are empty in our case. The relations (ii), (iii) and (iv) translate easily to relations (a), (b) and (c) in our theorem, respectively. The relations (d) are extra relations needed because we have taken a larger set of generators than in Brown's presentation. If $g_{E}\left(e_{0}\right)=e_{0}$, then the relation (d) is immediate. If $g_{E}\left(e_{0}\right) \neq e_{0}$, then $g_{E^{\prime}}\left(e_{0}\right)=e_{0}($ since $\gamma \notin K)$, and d) follows by reversing the roles of $g_{E}$ and $g_{E^{\prime}}$.

\section{No central elements}

In this section, our goal is to show that, in analogy to the case of surfaces of finite type, the baseleaf preserving mapping class group $\operatorname{MCG}(\mathcal{H})$ of the punctured solenoid has a trivial center. 
Proposition 5.1 A TLC tesselation of $\mathbf{D}$ is invariant under $\mathrm{PSL}_{2}(\mathbb{Z})$ if and only if it is the Farey tesselation $\tau_{*}$.

Proof We already know that $\mathrm{PSL}_{2}(\mathbb{Z})$ preserves the tesselation $\tau_{*}$ (indeed the group $\mathrm{PSL}_{2}(\mathbb{Z})$ is generated by hyperbolic reflections around the sides of the triangle $T \in \tau_{*}$ with vertices $(-1,1, i))$.

For the converse, recall that $\mathrm{PSL}_{2}(\mathbb{Z})$ contains order two elliptic elements with fixed points on each edge of $\tau_{*}$ and order three elliptic elements with fixed points at the center of each ideal complementary triangle of $\tau_{*}$. Let $T$ be a complementary triangle of $\tau$ containing the fixed point $a$ of an elliptic element $\gamma \in \mathrm{PSL}_{2}(\mathbb{Z})$ of order three. It is an exercise in elementary hyperbolic geometry to show that $a$ is the center of $T$.

Let $b$ be a fixed point of an elliptic involution $\gamma \in \operatorname{PSL}_{2}(\mathbb{Z})$. If $b$ is in the interior of a complementary triangle $T$ of $\tau$ then $\gamma(T) \neq T$ and $\gamma(T) \cap T \neq \varnothing$. Thus, the image of the boundary of $T$ under $\gamma$ intersects transversely the boundary of $T$. This is in contradiction to the assumption that $\gamma$ fixes $\tau$. It follows that $b$ must lie on an edge of $\tau$.

Let $a$ be the fixed point of an elliptic element of $\operatorname{PSL}_{2}(\mathbb{Z})$ of order three and let $b_{1}, b_{2}, b_{3}$ be fixed points of three elliptic involutions of $\operatorname{PSL}_{2}(\mathbb{Z})$ that are shortest distance to $a$ among all such involutions. Thus, $b_{1}, b_{2}, b_{3}$ lie on a hyperbolic circle centered at $a$. If $T$ is the ideal triangle in the complement of $\tau$ whose center is $a$, then the boundary sides of $T$ are tangent to this circle. Since $b_{1}, b_{2}, b_{3}$ must lie on edges of $\tau$, this implies that the boundary sides of $T$ are tangent at the points $b_{1}, b_{2}, b_{3}$. It follows that $T$ is a complementary triangle of $\tau_{*}$ as well. Since this is true for an arbitrary $T$, it follows that indeed $\tau=\tau_{*}$.

Theorem 1.3 The baseleaf preserving mapping class group $\operatorname{MCG}(\mathcal{H})$ of the punctured solenoid $\mathcal{H}$ has trivial center.

Proof Let $h \in \operatorname{MCG}(\mathcal{H})$ be a central element, so $h$ is a word in the generators of Theorem 1.2. For all $g \in \operatorname{MCG}(\mathcal{H})$, we must have $g \circ h \circ g^{-1}=h$. Let $\tau:=h\left(\tau_{*}\right)$. Taking $g \in \mathrm{PSL}_{2}(\mathbb{Z})$, we find $g \circ h\left(\tau_{*}\right)=h\left(\tau_{*}\right)$, ie, $g(\tau)=\tau$. By Proposition 5.1 above we conclude that $\tau=\tau_{*}$, ie, $h\left(\tau_{*}\right)=\tau_{*}$. But Penner-Šarić [14, Lemma 7.3] says that any homeomorphism $h: S^{1} \rightarrow S^{1}$ mapping $\tau_{*}$ to itself must be an element of $\mathrm{PSL}_{2}(\mathbb{Z})$. Therefore $h$ is in $\mathrm{PSL}_{2}(\mathbb{Z})$. Since $\mathrm{PSL}_{2}(\mathbb{Z})$ has trivial center, it follows that $h=i d$.

We consider the action of $\operatorname{MCG}(\mathcal{H})$ on the first barycentric subdivision $\mathcal{X}^{\prime}$ of $\mathcal{X}$. 
Proposition 5.2 The first barycentric subdivision $\mathcal{X}^{\prime}$ of $\mathcal{X}$ is a simplicial complex on which $\operatorname{MCG}(\mathcal{H})$ acts simplicially.

Proof Note that $\operatorname{MCG}(\mathcal{H})$ preserves cells of $\mathcal{X}$. An isotropy group of a vertex of $\mathcal{X}$ is a conjugate of $\mathrm{PSL}_{2}(\mathbb{Z})$. We showed that the isotropy group of an edge is either a finite index subgroup of $\mathrm{PSL}_{2}(\mathbb{Z})$ which preserves the orientation of the edge or it is generated by an element of $\operatorname{MCG}(\mathcal{H})$ which reverses the orientation of the edge and by a finite index subgroup of $\mathrm{PSL}_{2}(\mathbb{Z})$ which preserves the orientation of the edge. In the first case, each element of the isotropy group fixes each point on the edge. In the second case, an element either fixes each point of the edge or fixes the midpoint and reflects the endpoints of the edge.

Let $C$ be a coset two-cell with long edge given by a Whitehead move on TLC tesselation $\tau_{*}$ invariant under $K<\mathrm{PSL}_{2}(\mathbb{Z})$ along the orbit of $e \in \tau$ and with the short edges given by Whitehead moves invariant under a subgroup $H<K$. The isotropy subgroup of $C$ is a finite extension in $\operatorname{PSL}_{2}(\mathbb{Z})$ of $H$. In order to show that $\operatorname{MCG}(\mathcal{H})$ acts simplicially on the first barycentric subdivision of such a two-cell $C$, it is enough to show that the long edge cannot be mapped onto a short edge, and this is true because the rectangles in which change of diagonals for the Whitehead move occur must be mapped onto the rectangles on which change of diagonals occur. However, the two groups have different indexes in $\mathrm{PSL}_{2}(\mathbb{Z})$ which gives a contradiction, and the isotropy group $\Gamma(C)$ therefore acts by fixing each point in $C$. Since an arbitrary coset two-cell is the image of some $C$ as above, the same statement holds for an arbitrary coset two-cell.

Let $P$ be a pentagon two-cell based at $\tau_{*}$ obtained by Whitehead moves along $K\left\{e_{1}, e_{2}\right\}$. Thus, $P$ has a subgroup $K^{\prime}$, where $\mathrm{PSL}_{2}(\mathbb{Z})>K^{\prime}>K$, of its isotropy group $\Gamma(P)$ fixing each point of $P$. If $\Gamma(P) \neq K^{\prime}$, then it is generated by $K^{\prime}$ and a single element of $h \in \operatorname{MCG}(\mathcal{H})$ which maps the first edge onto the second. Since $h^{5}\left(\tau_{*}\right)=\tau_{*}$, we conclude $h^{5} \in \mathrm{PSL}_{2}(\mathbb{Z})$. Thus, $h^{5}$ conjugates a finite index subgroup of $\mathrm{PSL}_{2}(\mathbb{Z})$ onto itself (ie $h^{5}$ is mapping class-like) and therefore $h$ is mapping classlike (see Odden [11]). The map $h$ fixes the center of $P$ and rotates by the angle $2 \pi / 5$ the pentagon $P$. The situation for a pentagon not based at $\tau_{*}$ is the same.

Finally, suppose $Q$ is a square two-cell obtained by Whitehead moves along $K\left\{e_{1}, e_{2}\right\}$ then $\Gamma(Q)>K^{\prime}$, where $K<K^{\prime}<\operatorname{PSL}_{2}(\mathbb{Z})$. It is possible a priori that $\Gamma(Q) \neq K^{\prime}$, in which case the elements $h \in \Gamma(Q)-K^{\prime}$ permute edges of $Q$ and fix the center of $Q$.

We finally investigate the topological fundamental group $\pi_{1}(\mathcal{Y})$ of the quotient space $\mathcal{Y}=\mathcal{X} / \operatorname{MCG}(\mathcal{H})$. To begin, we describe a natural surjection $\phi: \operatorname{MCG}(\mathcal{H}) \rightarrow \pi_{1}(\mathcal{Y})$ 
as follows. Denote by $\Pi: \mathcal{X} \rightarrow \mathcal{Y}$ the quotient map, let $h \in \operatorname{MCG}(\mathcal{H})$ be arbitrary, and define $\tau=h\left(\tau_{*}\right)$. Let $\gamma$ be an edge-path between $\tau_{*}$ and $\tau$ in $\mathcal{X}$. Define

$$
\phi(h):=[\Pi(\gamma)]
$$

where $[\Pi(\gamma)]$ is the homotopy class of the closed curve $\Pi(\gamma)$ based at $\Pi\left(\tau_{*}\right)$, ie, $[\Pi(\gamma)] \in \pi_{1}\left(\mathcal{Y}, \Pi\left(\tau_{*}\right)\right)=\pi_{1}(\mathcal{Y})$. It is a standard fact that $\phi$ is a well-defined and surjective homomorphism.

Let $\mathcal{N}$ be the group generated by the isotropy subgroups of all vertices of $\mathcal{X}^{\prime}$, where $\mathcal{X}^{\prime}$ denotes the first barycentric subdivision of $\mathcal{X}$, so $\mathcal{N}$ is normal in $\operatorname{MCG}(\mathcal{H})$. In fact, $\mathcal{N}$ is generated by all conjugates of $\mathrm{PSL}_{2}(\mathbb{Z})$ and by the isotropy groups of edges and two-cells of $\mathcal{X}$. The isotropy group of an edge in $\mathcal{X}$ fixes the center of the edge and therefore belongs to the isotropy group of a vertex in $\mathcal{X}^{\prime}$, and likewise the isotropy group of a two-cell in $\mathcal{X}$ fixes a vertex of $\mathcal{X}^{\prime}$. Moreover, any element of $\operatorname{MCG}(\mathcal{H})$ which fixes a point in $\mathcal{X}^{\prime}$ fixes a point in $\mathcal{X}$.

At this point we use the following result proved by Armstrong in [1].

Theorem (Armstrong [1]) Let $G$ act simplicially on a connected and simply connected complex $K$, and let $H$ be the normal subgroup generated by those elements of $G$ which leave some point fixed. Then the fundamental group of the orbit space $|K| / G$ is isomorphic to $G / H$.

Together with our Proposition 5.2, the above theorem yields the next result.

Theorem 1.4 The topological fundamental group of $\mathcal{Y}=\mathcal{X} / \operatorname{MCG}(\mathcal{H})$ satisfies

$$
\pi_{1}(\mathcal{Y})=\operatorname{MCG}(\mathcal{H}) / \mathcal{N}
$$

where $\mathcal{N}<\operatorname{MCG}(\mathcal{H})$ is generated by the isotropy groups of vertices, edges and two-cells of $\mathcal{X}$.

By our discussion above, each element of $\operatorname{MCG}(\mathcal{H})$ which fixes a cell in $\mathcal{X}$ is mapping class-like, meaning that it conjugates a finite index subgroup of $G$ onto itself. Therefore $\mathcal{N}$ is generated by some mapping class-like elements. We pose the following question.

Question Is $\mathcal{N}$ equal to the normal subgroup of $\operatorname{MCG}(\mathcal{H})$ generated by all mapping class-like elements? 


\section{References}

[1] M A Armstrong, On the fundamental group of an orbit space, Proc. Cambridge Philos. Soc. 61 (1965) 639-646 MR0187244

[2] K S Brown, Presentations for groups acting on simply-connected complexes, J. Pure Appl. Algebra 32 (1984) 1-10 MR739633

[3] A Douady, C J Earle, Conformally natural extension of homeomorphisms of the circle, Acta Math. 157 (1986) 23-48 MR857678

[4] D B A Epstein, R C Penner, Euclidean decompositions of noncompact hyperbolic manifolds, J. Differential Geom. 27 (1988) 67-80 MR918457

[5] J L Harer, The virtual cohomological dimension of the mapping class group of an orientable surface, Invent. Math. 84 (1986) 157-176 MR830043

[6] A Hatcher, On triangulations of surfaces, Topology Appl. 40 (1991) 189-194 MR1123262

[7] A Hatcher, W Thurston, A presentation for the mapping class group of a closed orientable surface, Topology 19 (1980) 221-237 MR579573

[8] N V Ivanov, Automorphisms of complexes of curves and of Teichmüller spaces, from: "Progress in knot theory and related topics", Travaux en Cours 56, Hermann, Paris (1997) 113-120 MR1603146

[9] S Nag, D Sullivan, Teichmüller theory and the universal period mapping via quantum calculus and the $H^{1 / 2}$ space on the circle, Osaka J. Math. 32 (1995) 1-34 MR1323099

[10] C Odden, Virtual automorphism group of the fundamental group of a closed surface, $\mathrm{PhD}$ thesis, Duke University, Durham (1997)

[11] C Odden, The baseleaf preserving mapping class group of the universal hyperbolic solenoid, Trans. Amer. Math. Soc. 357 (2005) 1829-1858 MR2115078

[12] R C Penner, The decorated Teichmüller space of punctured surfaces, Comm. Math. Phys. 113 (1987) 299-339 MR919235

[13] R C Penner, Universal constructions in Teichmüller theory, Adv. Math. 98 (1993) 143-215 MR1213724

[14] R C Penner, D Šarić, Teichmüller theory of the punctured solenoid IMS preprint Available at http:/www.math.sunysb.edu/ saric/

[15] D Sullivan, Linking the universalities of Milnor-Thurston, Feigenbaum and AhlforsBers, from: "Topological methods in modern mathematics (Stony Brook, NY, 1991)", Publish or Perish, Houston, TX (1993) 543-564 MR1215976 
Institute for Mathematical Sciences, Stony Brook University,

Stony Brook, NY 11794-3660

Departments of Mathematics and Physics/Astronomy, University of Southern California, Los Angeles, CA 90089

Institute for Mathematical Sciences, Stony Brook University,

Stony Brook, NY 11794-3660,

Current address: Department of Mathematics, Queens College of CUNY, 65-30 Kissena Blvd, Flushing, NY 11367

bonnot@math.sunysb.edu, rpenner@usc.edu, dragomir.saric@qc.cuny.edu http://www.math.sunysb.edu/ bonnot/, http://www-rcf.usc.edu/ rpenner/, http://www . math. sunysb.edu/ saric/

Received: 6 November $2006 \quad$ Revised: 29 June 2007

Algebraic $\&$ Geometric Topology, Volume 7 (2007) 